ESAIM: PROCEEDINGS AND SURVEYS, 2020, Vol. 68, p. 73-96

Hervé Cardot \& Pierre Calka

\title{
SOME RECENT ADVANCES FOR LIMIT THEOREMS
}

\author{
Benjamin Arras ${ }^{1}$, Jean-Christophe Breton ${ }^{2}$, Aurelia Deshayes ${ }^{3}$, Olivier \\ DURIEU $^{4}$ AND RAPHAËL LACHIÈZE-REY ${ }^{5}$
}

\begin{abstract}
We present some recent developments for limit theorems in probability theory, illustrating the variety of this field of activity. The recent results we discuss range from Stein's method, as well as for infinitely divisible distributions as applications of this method in stochastic geometry, to asymptotics for some discrete models. They deal with rates of convergence, functional convergences for correlated random walks and shape theorems for growth models.

Résumé. On présente quelques résultats récents dans le domaine des théorèmes limites en probabilités illustrant la variété de ce champ d'activité. Les résultats récents que nous discutons vont de la méthode de Stein aussi bien pour des lois infiniment divisibles que pour ses applications en géométrie stochastique à des asymptotiques pour quelques modèles discrets. Ils traitent de vitesses de convergence, de convergences fonctionnelles pour des marches aléatoires corrélées et de théorèmes de forme pour des modèles de croissance.
\end{abstract}

\section{INTRODUCTION}

Limit theorems form a branch of probability theory whose activity still has a great influence on other probabilistic fields since most probabilistic analyses are usually completed by a limit theorem. They make emerge some characteristic or universal behaviours from a stochastic context. Initiated with the celebrated law of large numbers (LLN) and the central limit theorem (CLT) for the asymptotic behaviour of the sum $S_{n}=\sum_{i=1}^{n} X_{i}$ of independent and identically distributed (iid) random variables $X_{i}, i \geq 1$, limit theorems have been developed in various directions ranging from iid to dependent random variables, from central to non-central theorems, from convergence in distribution for random variables to functional convergence for stochastic processes or from mere limits to quantification of the convergences to name but a few developments. They have numerous applications starting from statistical properties of related estimators to stochastic approximation in various models. The aim of this survey article is to highlight some various recent developments in some selected directions: sharp rates of convergence in limit theorems with Stein's method, functional limit theorems under dependence, and shape theorems for discrete growth models.

${ }^{1}$ Univ. Lille, CNRS, UMR 8524 - Laboratoire Paul Painlevé, F-59000 Lille, France. E-mail address: arrasbenjamin@gmail.com

${ }^{2}$ Univ Rennes, CNRS, IRMAR-UMR 6625, F-35000 Rennes, France. E-mail address: jean-christophe.breton@univ-rennes1.fr

3 Learning, Data \& Robotics Lab - ESIEA, Paris, France and Université Paris-Est, Laboratoire d'Analyse et de Mathématiques Appliquées-UMR 8050, UPEM, UPEC, CNRS, F-94010, Créteil, France. E-mail address: deshayes@esiea.fr

${ }^{4}$ Institut Denis Poisson-UMR 7013, Université de Tours, Parc de Grandmont, 37200 Tours, France. E-mail address: olivier.durieu@univtours.fr

${ }^{5}$ MAP5-UMR 8145, Université Paris Descartes, Sorbonne Paris Cité, France. E-mail address: raphael.lachieze-rey@parisdescartes.fr

(C) EDP Sciences, SMAI 2020

This is an Open Access article distributed under the terms of the Creative Commons Attribution License (http://creativecommons.org/licenses/by/4.0), which permits unrestricted use, distribution, and reproduction in any medium, provided the original work is properly cited. 
Originally, usual limit theorems deal with the convergence in distribution of the partial sums $S_{n}, n \geq 1$, of a sequence of random variables $\left(X_{i}\right)_{i \geq 1}$ properly centered and normalized

$$
\frac{S_{n}-b_{n}}{c_{n}} \Longrightarrow Z, \quad n \rightarrow+\infty
$$

where $Z$ is a non-degenerate random variable. When the $X_{i}$ 's are $i i d$ with finite non-zero variance, $b_{n}=\mathbb{E}\left[X_{1}\right] n$, $c_{n}=\sqrt{\operatorname{Var}\left(X_{1}\right) n}$, then $Z \sim \mathcal{N}(0,1)$ and the convergence (1) is the usual CLT. More generally, a fundamental theorem of Khintchine (see, e.g., [78]) shows that for an array $\left(X_{n, i}\right)_{1 \leq i \leq k_{n}}$ of random variables independent within each rows of sizes $k_{n} \rightarrow+\infty$ when $n \rightarrow+\infty$, the limit theorem (1) holds true for the sum $S_{n}=\sum_{i=1}^{k_{n}} X_{n, i}$ when the random variables $X_{n, i}, 1 \leq i \leq k_{n}, n \geq 1$, are infinitely small in the sense that for all $\varepsilon>0$ :

$$
\lim _{n \rightarrow+\infty} \max _{1 \leq i \leq k_{n}} \mathbb{P}\left(\left|X_{n, i}\right| \geq \varepsilon\right)=0 .
$$

The possible limits in (1) then coincide with the set of infinitely divisible distributions, see Theorem 3.1 in [96] to which we refer also for a general account on limit theorems for sums of independent random variables.

Non-independent random variables $\left(X_{i}\right)_{i \geq 1}$ can also be considered in (1) and the limit depends on the type of dependence exhibited by the $X_{i}$ 's. As an illustration of limit theorem for non-independent random variables, consider the $q$-Hermite power variations of a fractional Brownian motion $\left(B_{t}^{(H)}\right)_{t \geq 0}$ with Hurst index $H \in(0,1)$ (see (29) for a definition): take the non-independent random variables $X_{i}=H_{q}\left(B_{i}^{(H)}-B_{i-1}^{(H)}\right), i \geq 1$, where $H_{q}(x)=(-1)^{q} e^{x^{2} / 2} \frac{d^{q} e^{-x^{2} / 2}}{d x^{q}}$ is the Hermite polynomial with degree $q$, and consider $S_{n}=\sum_{i=1}^{n} X_{i}$. In this case, we have from $[21,44,66,118]$ :

- for $0<H<1-1 /(2 q)$, then

$$
\frac{S_{n}}{\sigma_{q, H} \sqrt{n}} \Longrightarrow \mathcal{N}(0,1)
$$

- for $H=1-1 /(2 q)$, then

$$
\frac{S_{n}}{\sigma_{q, H} \sqrt{n \log n}} \Longrightarrow \mathcal{N}(0,1)
$$

- for $1-1 /(2 q)<H<1$, then

$$
\frac{S_{n}}{n^{1-q(1-H)}} \Longrightarrow Z
$$

where $Z$ is some Hermite random variable and $\sigma_{q, H}$ above is some explicit constant.

Beyond the original case of sums of random variables, limit theorems have been obtained for numerous kinds of random variables. For instance, [19] deals with functionals on a random balls model with interactions and the limit theorems therein give a macroscopic insight into the model with asymptotic distributions as diverse as Gaussian, Poissonian or stable. In the sequel, we shall present such limit theorems for some specific random variables that are not sum of random variables, see Section 1.3 and Section 3.

An active line of research for limit theorems consists in giving rates of convergence. For instance, the celebrated Berry-Esseen theorem gives a rate of convergence in $1 / \sqrt{n}$ for the CLT in the Kolmogorov distance whenever the $X_{i}$ 's have a finite third moment: for centered iid random variables $\left(X_{i}\right)_{i \geq 1}$ with unit variance

$$
\sup _{x \in \mathbb{R}}\left|\mathbb{P}\left(S_{n} / \sqrt{n} \leq x\right)-\mathbb{P}(N \leq x)\right| \leq C \frac{\mathbb{E}\left[\left|X_{1}\right|^{3}\right]}{\sqrt{n}}
$$

where $N \sim \mathcal{N}(0,1)$ and $C$ is a (universal) constant. A fruitful strategy to obtain such rates of convergence is Stein's method which produces sharp upper bounds on distances between probability measures. For instance in [29], using this strategy Chen and Ho prove (5). This method is used also in [89] to give rates of convergence 
for (2), (3), see also [20] for (4). In the sequel, we shall visit some recent developments for Stein's method in Section 1.

Another kind of limit theorems deals with functional counterparts of (1) where stochastic processes are considered instead of random variables. The most famous such limit theorem is Donsker's invariance principle: this functional counterpart of the CLT states that the piecewise constant process $\left(S_{\lfloor n t\rfloor}\right)_{t \in[0,1]}$ associated to the partial sum $S_{n}$ of iid square integrable random variables $\left(X_{i}\right)_{i \geq 1}$, say centered with unit variance, satisfies

$$
\left(\frac{S_{\lfloor n t\rfloor}}{\sqrt{n}}\right)_{t \in[0,1]} \stackrel{J_{1}}{\Longrightarrow}\left(B_{t}\right)_{t \in[0,1]}
$$

where the weak convergence above holds in the Skorohod space $\mathbb{D}([0,1])$ of right-continuous functions with left limits on $[0,1]$ equipped with Skorohod's $J_{1}$-topology and $\left(B_{t}\right)_{t \in[0,1]}$ is a usual Brownian motion on [0,1], see e.g. [11]. Here, and in the sequel, the notation $\lfloor x\rfloor$ stands for the integer part of $x \in \mathbb{R}$. Extensions of this result in presence of dependence will be discussed in Section 2.

The paper is organized as follows. Section 1 deals with the quantification of limit theorems with Stein's method: after a short presentation of this method in Section 1.1, we present in Section 1.2 some recent Stein bounds obtained in [2] where the method is developed for infinitely divisible distributions with finite first moment. Next in Section 1.3, we illustrate the application of Stein's method in combination with Malliavin calculus to obtain rate of convergence for CLT for some Poissonian functionals of stochastic geometry, in particular optimal such bounds are obtained for the functional of nearest Poissonian neighbour and for some characteristic quantities of Poissonian convex hull, see [81]. In Section 2, we present some recent generalizations on Donsker's invariance principle (6) in [10,47] both for correlated random walks (Section 2.1) and for correlated random fields (Section 2.2). The correlation considered derives from a random graph structure in the set of indices inspired from Hammond and Sheffield in [71]. Finally in Section 3, we deal with another discrete model presenting the propagation of an infection on a discrete graph; in this case, we are interested in the set of infected vertices and the limit theorems describe the asymptotic shape of such a set. In particular, we deal with first passage percolation model from [32] in Section 3.1, with the contact process from [48] in Section 3.2 and with non-attractive model such as Fredrickson-Andersen one spin facilitated model in dimension one from [14] in Section 3.3.

\section{STEIN'S METHOD AND QUANTIFICATION OF LIMIT THEOREMS}

\subsection{Stein's method}

Stein's method is a powerful tool to quantify weak limit theorems. It originates from Stein's characterization of the Gaussian standard distribution, i.e. $N \sim \mathcal{N}(0,1)$ if and only if for all functions $f: \mathbb{R} \rightarrow \mathbb{R}$, bounded and Lipschitz on $\mathbb{R}$, we have

$$
\mathbb{E}\left[N f(N)-f^{\prime}(N)\right]=0 \text {. }
$$

Developed in the Gaussian setting by Stein in $[115,116]$, the method relies on the corresponding Stein equation in function $f: \mathbb{R} \rightarrow \mathbb{R}$

$$
f^{\prime}(x)-x f(x)=h(x)-\mathbb{E}[h(N)], \quad x \in \mathbb{R} .
$$

Alternatively, it is said that the operator $L f(x)=f^{\prime}(x)-x f(x)$ is the Gaussian Stein characterizing operator. Moreover, when $h$ is in $\mathcal{H}$ the set of Lipschitz functions $h: \mathbb{R} \rightarrow \mathbb{R}$ with $\|h\|_{L}:=\sup _{x \neq y}|h(x)-h(y)| /|x-y| \leq 1$, it is shown in [24, Lemma 4.2] or [116] that the solution $f_{h}$ of Stein equation (8) is in $\mathcal{F}_{\mathcal{H}}=\left\{f \in C^{2}(\mathbb{R})\right.$ : $\left.\left\|f^{\prime}\right\|_{\infty} \leq 1,\left\|f^{\prime \prime}\right\|_{\infty} \leq 2\right\}$ where $C^{2}(\mathbb{R})$ is the space of twice differentiable function $g: \mathbb{R} \rightarrow \mathbb{R}$ with continuous twice derivative and, for any $g: \mathbb{R} \rightarrow \mathbb{R}$, we set $\|g\|_{\infty}:=\sup _{x \in \mathbb{R}}|g(x)|$. As a consequence, we derive the following Stein bound for the Wasserstein distance $d_{W}$

$$
d_{W}(X, N):=\sup _{h \in \mathcal{H}}|\mathbb{E}[h(X)]-\mathbb{E}[h(N)]| \leq \sup _{f \in \mathcal{F}_{\mathcal{H}}}\left|\mathbb{E}\left[X f(X)-f^{\prime}(X)\right]\right|
$$


Note that here we focus on Stein bound for the Wasserstein distance $d_{W}$ but other distances can be considered with other choices of test functions $\mathcal{H}$ like the smooth Wasserstein-2 distance $d_{W_{2}}$ in Section 1.2.

Alternatively, a generator approach has been used similarly by Barbour in [5] and Götze in [68] replacing (7) by the observation that $\mathcal{N}(0,1)$ is the stationary distribution of an Ornstein-Uhlenbeck $(\mathrm{OU})$ process $\left(U_{t}\right)_{t \geq 0}$, solution of $d U_{t}=-U_{t} d t+\sqrt{2} d B_{t}$ with $U_{0}=x$ and where $\left(B_{t}\right)_{t \geq 0}$ is a standard Brownian motion. Introducing the OU-generator $\mathcal{A} h(x)=-x h^{\prime}(x)+h^{\prime \prime}(x)$, the corresponding OU-semigroup $P_{t} h(x)=\mathbb{E}\left[h\left(U_{t}\right) \mid U_{0}=x\right]$ writes (formally) $P_{t}=\exp (t \mathcal{A})$ and satisfies $\frac{d}{d t} P_{t}=\mathcal{A} P_{t}$. Stein equation (8) is then replaced by

$$
\mathbb{E}[h(N)]-h(x)=\int_{0}^{+\infty} \frac{d}{d t} P_{t} h(x) d t=\int_{0}^{+\infty} \mathcal{A} P_{t} h(x) d t, \quad x \in \mathbb{R},
$$

from which we derive for regular enough function $h$

$$
|\mathbb{E}[h(N)]-\mathbb{E}[h(X)]|=\left|\mathbb{E}\left[\int_{0}^{+\infty} \mathcal{A} P_{t} h(X) d t\right]\right|,
$$

see [35]. Various strategies complete this approach to quantify the bounds (9) or (11) by exploiting the special structure of $X$, for instance when typically, as in (1), $X$ is a centered and normalized partial sums of random variables. Among these strategies, we can mention the fruitful combination with Malliavin calculus initiated by Nourdin and Peccati in [89]. We shall illustrate such a combination in Section 1.3 in a context of Poissonian functional with applications to recent asymptotic normality results in [81] in stochastic geometry.

After its Gaussian implementation, this method has been extended by Chen for Poisson distribution $\mathcal{P}(\lambda)$ in $[28]$ based on the Poissonian counterpart of $(7): X \sim \mathcal{P}(\lambda)$ with parameter $\lambda>0$ if and only if for all bounded function $f: \mathbb{N} \rightarrow \mathbb{N}$ :

$$
\mathbb{E}[X f(X)-\lambda f(X+1)]=0
$$

Since then, the method has been developed for numerous probability distributions both in univariate and multivariate contexts producing quantitative bounds for limit theorems (1) with corresponding asymptotic distributions. Let us mention the following series of papers (together with their associated target limiting laws): for exponential and Laplace approximations $[27,55,93,98]$, for gamma and chi-squared approximations [43, 64, 85, 97], for Compound Poisson approximation [6], for negative binomial approximation [22], for beta approximation [42,67], for semicircular approximation [69], for variance-gamma approximation [58, 62], for two-sided Maxwell approximation [86] and for symmetric $\alpha$-stable approximation [122].

It is worthwhile to note that this method appears to be a powerful substitute to the usual Fourier transform approach originating from the fundamental work of Esseen [54]. Indeed, Stein's method can be effective in the presence of dependency and can deal with more complex sequential structures than sums of iid summands. Moreover, several interactions with other domains of probability theory have been identified and as such have revealed the fecund nature of the method, see for instance the work of Chatterjee [24, 25]. For standard references on the method as well as for its many ramifications we refer the reader to the books and surveys $[7,26,30,83,90,109]$.

An active line of work for this method focuses now on finding Stein characterizing operators for new probability distributions for which the usual Stein's method framework seems difficult to implement. In this direction, Stein characterizing operators have been obtained for linear combination of independent gamma random variables [1], for product type distributions $[60,61,65]$ as well as for some anecdotal distributions [59, 63]. In Section 1.2, we show how the Stein methodology has recently been developed in [2] for infinitely divisible target probability measures, such distributions encompass a large number of probability measures on $\mathbb{R}$.

\subsection{Stein's method for infinitely divisible distribution}

Real infinitely divisible (ID) distributions $I D\left(b, \sigma^{2}, \nu\right)$ with $b \in \mathbb{R}, \sigma^{2} \geq 0$ and Lévy measure $\nu$ appear as the natural limiting distributions in limit theorem (1) for sums of independent random variables which are uniformly 
asymptotically negligible at infinity. For a standard reference on ID distributions, the reader is referred to [112]. As mentionned above, Stein's method has been implemented only for a few ID distributions until very recently with [2]. Indeed in a general ID setting, [2] develops a Stein methodology under a first moment assumption producing quantitative versions of weak non-central limit theorems (1).

Let us describe the cornerstone of the approach followed in [2] to enforce a Stein's method for a subclass of ID distributions, without Gaussian component to simplify the presentation (i.e. $\sigma^{2}=0$ ). This approach relies on a characterization result identifying a Stein operator for every ID distribution with finite first moment like in (7) for Gaussian or (12) for Poisson distribution: for a random variable $X$ with finite first moment, $X \sim I D(b, 0, \nu)$ if and only if

$$
\mathbb{E}\left[X f(X)-b f(X)-\int_{\mathbb{R}}\left(f(X+u)-f(X) \mathbf{1}_{\{|u| \leq 1\}}\right) u \nu(d u)\right]=0,
$$

for all bounded and Lipschitz function $f: \mathbb{R} \rightarrow \mathbb{R}$, see Theorem 3.1 in [2]. Observe that (13) reduces to (12) for $I D\left(\lambda, 0, \lambda \delta_{1}\right)=\mathcal{P}(\lambda)$. This ID characterizing Stein operator (13) is based on the following covariance identity for ID random vectors established in [73] and from which many crucial results for ID distributions follow. Let $\left(X_{z}, Y_{z}\right)$ be a two-dimensional ID random vector with characteristic function $(\varphi(t) \varphi(s))^{1-z} \varphi(t+s)^{z}$, for any $z \in[0,1]$, where $\varphi$ stands for the characteristic function of $X$. Then, for two bounded Lipschitz functions $f, g$,

$$
\operatorname{Cov}(f(X), g(X))=\int_{0}^{1} \mathbb{E}_{z}\left[\int_{-\infty}^{+\infty}\left(f\left(X_{z}+u\right)-f\left(X_{z}\right)\right)\left(g\left(Y_{z}+u\right)-g\left(Y_{z}\right)\right) \nu(d u)\right] d z .
$$

Based on (13), it is natural to infer the following non-local equation as a Stein equation for a random variable $X \sim I D(b, 0, \nu)$ with finite first moment like (8) derives from (7):

$$
(b-x) f_{h}(x)+\int_{\mathbb{R}}\left(f_{h}(x+u)-f_{h}(x) \mathbf{1}_{\{|u| \leq 1\}}\right) u \nu(d u)=h(x)-\mathbb{E}[h(X)], \quad x \in \mathbb{R},
$$

where $h$ is some appropriate test function on $\mathbb{R}$. As developed in Chapter 5 of [2], this intuition leads indeed to concrete results regarding Stein's method that we present below for self-decomposable distributions with finite first moment. This method is an extension of the generator approach introduced by Barbour in [5] and Götze in [68] in the Gaussian setting and summarized in (10)-(11). First, recall that a random variable $X$ is self-decomposable if, for any $c \in(0,1)$, there exists a random variable $X_{c}$ independent of $X$, such that $X \stackrel{d}{=} c X+X_{c}$, where $\stackrel{d}{=}$ stands for equality in distribution. In particular, self-decomposable distributions are infinitely divisible.

To fully understand how the generator approach can be implemented for self-decomposable distributions, let us first describe the decomposability properties of a self-decomposable distribution (for further details see e.g. [75]). For a probability measure $\mu$ on $(\mathbb{R}, \mathscr{B}(\mathbb{R}))$, set $D(\mu)=\left\{c \in(0,1): \mu=\Theta_{c}(\mu) * \mu_{c}\right.$ for some real probability measure $\left.\mu_{c}\right\}$ its set of decomposability where, for any $c \in(0,1), \Theta_{c}$ denotes the scaling operator acting on probability measures and defined by

$$
\int_{\mathbb{R}} h(x) \Theta_{c}(\mu)(d x)=\int_{\mathbb{R}} h(c x) \mu(d x),
$$

for all $h$ bounded real measurable function. For any self-decomposable real probability measures $\mu$, by definition, we have $D(\mu)=(0,1)$ and it is a semigroup for the standard multiplication between scalars. In particular, it contains the one-parameter semigroup $\left(T_{t}\right)_{t \geq 0}$ defined by

$$
T_{t}(x):=e^{-t} x, \quad x \in(0,1), \quad t \geq 0,
$$

and we still denote $T_{t}$ the associated operator $T_{t}=\Theta_{e^{-t}}$ on probability measures. Then, for a self-decomposable probability measure $\mu$ on $(\mathbb{R}, \mathscr{B}(\mathbb{R}))$, we define the following family $\left(\mu_{t}\right)_{t>0}$ of probability measures through their 
characteristic functions

$$
\varphi_{t}(y):=\int_{\mathbb{R}} e^{i x y} \mu_{t}(d x)=\frac{\varphi(y)}{\varphi\left(e^{-t} y\right)}, \quad y \in \mathbb{R}, \quad t \geq 0,
$$

where $\varphi$ stands for the characteristic function of $\mu$. Note in particular that this family of probability measures satisfies the following measure-valued cocycle equation

$$
\mu_{t+s}=\mu_{t} * T_{t}\left(\mu_{s}\right), \quad s, t \geq 0,
$$

which is easily seen via characteristic functions since for $y \in \mathbb{R}$ and $s, t \geq 0$

$$
\varphi_{\mu_{t} * T_{t}\left(\mu_{s}\right)}(y)=\frac{\varphi(y)}{\varphi\left(e^{-t} y\right)} \frac{\varphi\left(e^{-t} y\right)}{\varphi\left(e^{-(t+s)} y\right)}=\varphi_{t+s}(y)
$$

and $\mu$ is solution of $\mu=T_{t} \mu * \mu_{t}$. The convolution identity (14) implies that the family of operators $\left(P_{t}\right)_{t \geq 0}$ defined, for all $h \in C_{b}(\mathbb{R})$ (the space of bounded continuous functions on $\mathbb{R}$ ), by

$$
P_{t} h(x)=\int_{\mathbb{R}} h\left(T_{t}(x)+y\right) \mu_{t}(d y), \quad x \in \mathbb{R}, t \geq 0
$$

enjoys the semigroup property on $C_{b}(\mathbb{R})$. In particular, this semigroup of operators satisfies the following invariance and ergodicity properties

$$
\int_{\mathbb{R}} P_{t} h(x) \mu(d x)=\int_{\mathbb{R}} h(x) \mu(d x), \quad \lim _{t \rightarrow+\infty} P_{t} h(x)=\int_{\mathbb{R}} h(x) \mu(d x) .
$$

As usual, the next step is to identify the generator of this semigroup $\left(P_{t}\right)_{t \geq 0}$ in order to infer a relevant Stein equation for the target probability measure $\mu$. Under a first moment constraint, using Fourier duality to express the semigroup $\left(P_{t}\right)_{t \geq 0}$ in a relevant form on $\mathcal{S}(\mathbb{R})$, the Schwartz space of infinitely differentiable functions on $\mathbb{R}$ with rapid decrease at $\pm \infty$, it is possible to identify the pointwise generator of the semigroup $\left(P_{t}\right)_{t \geq 0}$ on $\mathcal{S}(\mathbb{R})$ as the pseudo-differential operator whose symbol is given by

$$
q(x, y)=(i y)\left(-x+\mathbb{E}[X]+\int_{\mathbb{R}}\left(e^{i u y}-1\right) u \nu(d u)\right), \quad x \in \mathbb{R}, y \in \mathbb{R},
$$

where $\nu$ is the Lévy measure of the self-decomposable probability measure $\mu$ and $X \sim \mu$. Then, the Stein equation associated with $X$ writes, for all $h \in C_{c}^{\infty}(\mathbb{R})$ such that $\|h\|_{\infty} \leq 1,\left\|h^{\prime}\right\|_{\infty} \leq 1$ and $\left\|h^{\prime \prime}\right\|_{\infty} \leq 1$ :

$$
h(x)-\mathbb{E}[h(X)]=(\mathbb{E}[X]-x) f_{h}^{\prime}(x)+\int_{\mathbb{R}}\left(f_{h}^{\prime}(x+u)-f_{h}^{\prime}(x)\right) u \nu(d u), \quad x \in \mathbb{R},
$$

where $C_{c}^{\infty}(\mathbb{R})$ is the space of real infinitely differentiable functions with compact support. At this point, the classical machinery of the generator approach for Stein's method can be performed in the general setting of self-decomposable probability measure with finite first moment. Namely, the solution $f_{h}$ of the Stein equation (16) is given by

$$
f_{h}(x)=-\int_{0}^{+\infty}\left(P_{t} h(x)-\mathbb{E}[h(X)]\right) d t, \quad x \in \mathbb{R} .
$$

In particular, since $\|h\|_{\infty} \leq 1,\left\|h^{\prime}\right\|_{\infty} \leq 1$ and $\left\|h^{\prime \prime}\right\|_{\infty} \leq 1$ and since the commutation relation holds

$$
\frac{d}{d x}\left(P_{t} h(x)\right)=e^{-t} P_{t}\left(h^{\prime}\right)(x)
$$


for all $t \geq 0$ and for all $x \in \mathbb{R}$, Stein magic factors for the function $f_{h}$ are $\left\|f_{h}^{\prime}\right\|_{\infty} \leq 1$ and $\left\|f_{h}^{\prime \prime}\right\|_{\infty} \leq \frac{1}{2}$. Like for (9), Stein equation (16) and the previous bounds entail the general Stein bound when $X$ is self-decomposable with finite first moment in term of the smooth Wasserstein-2 distance $d_{W_{2}}$ :

$$
\begin{aligned}
d_{W_{2}}(Y, X) & :=\sup _{\substack{h \in C_{c}^{\infty}(\mathbb{R}),\|h\|_{\infty} \leq 1 \\
\left\|h^{\prime}\right\|_{\infty} \leq 1,\left\|h^{\prime \prime}\right\|_{\infty} \leq 1}}|\mathbb{E}[h(X)]-\mathbb{E}[h(Y)]| \\
& \leq \sup _{\substack{f \in C^{2}(\mathbb{R}),\left\|f^{\prime}\right\| \infty \leq 1,\left\|f^{\prime \prime}\right\|_{\infty} \leq 1 / 2}} \mid \mathbb{E}\left[(\mathbb{E}[X]-Y) f^{\prime}(Y)+\int_{\mathbb{R}}\left(f^{\prime}(Y+u)-f^{\prime}(Y)\right) u \nu(d u)\right] .
\end{aligned}
$$

Let us illustrate now on the example of the generalized Dickman distribution the interest of the new derived Stein equation (16), Stein solution (17) and relative bound (18). Recall that the generalized Dickman distribution with parameter $\theta>0$ is the self-decomposable probability measure $I D\left(\theta, 0, \theta \mathbf{1}_{(0,1)}(u) d u / u\right)$ defined through the following Lévy-Khintchine representation of its characteristic function

$$
\varphi(y)=\exp \left(\theta \int_{0}^{1} \frac{e^{i u y}-1}{u} d u\right), \quad y \in \mathbb{R} .
$$

The generalized Dickman distribution, introduced by Dickman in 1930 [41] to describe the largest prime factor of a random integer appears in number theory (see e.g. [23]) and in some type of combinatorial structures (see [3]).

Theorem 1.1. Let $X_{\theta}$ be a generalized Dickman distributed random variable with parameter $\theta>0$, let also $\left(Y_{k}\right)_{k \geq 1}$ be a sequence of independent random variables such that, for all $k \geq 1, Y_{k}$ is Poisson distributed with parameter $\theta / k$ and let $S_{n}=\frac{1}{n} \sum_{k=1}^{n} k Y_{k}$ for $n \geq 1$. Then, for all $n \geq 1$,

$$
d_{W_{2}}\left(S_{n}, X_{\theta}\right) \leq \frac{\theta}{4 n} .
$$

Proof: The previous general bound (18) writes

$$
d_{W_{2}}\left(S_{n}, X_{\theta}\right) \leq \sup _{\substack{f \in C^{2}(\mathbb{R}),\left\|f^{\prime}\right\| \infty \leq 1,\left\|f^{\prime \prime}\right\|_{\infty} \leq 1 / 2}}\left|\mathbb{E}\left[\left(\mathbb{E}\left[X_{\theta}\right]-S_{n}\right) f^{\prime}\left(S_{n}\right)+\theta \int_{0}^{1}\left(f^{\prime}\left(S_{n}+u\right)-f^{\prime}\left(S_{n}\right)\right) d u\right]\right| .
$$

In order to estimate the right-hand side of (19), let $f \in C^{2}(\mathbb{R})$ with $\left\|f^{\prime}\right\|_{\infty} \leq 1,\left\|f^{\prime \prime}\right\|_{\infty} \leq 1 / 2$ and note that $\mathbb{E}\left[S_{n}\right]=\mathbb{E}\left[X_{\theta}\right]=\theta$. Next, set $S_{n, k}=S_{n}-k Y_{k} / n$ for all $n \geq 1$ and $1 \leq k \leq n$. Then, for all $n \geq 1$

$$
\mathbb{E}\left[S_{n} f^{\prime}\left(S_{n}\right)\right]=\frac{1}{n} \sum_{k=1}^{n} k \mathbb{E}\left[Y_{k} f^{\prime}\left(S_{n, k}+\frac{k}{n} Y_{k}\right)\right] .
$$

Since $Y_{k}$ is Poisson distributed with parameter $\theta / k$ and is independent from $S_{n, k}$, the Chen-Stein identity (12) ensures

$$
\mathbb{E}\left[Y_{k} f^{\prime}\left(S_{n, k}+\frac{k}{n} Y_{k}\right)\right]=\mathbb{E}\left[\mathbb{E}\left[Y_{k} f^{\prime}\left(S_{n, k}+\frac{k}{n} Y_{k}\right) \mid S_{n, k}\right]\right]=\mathbb{E}\left[\frac{\theta}{k} \mathbb{E}\left[f^{\prime}\left(S_{n, k}+\frac{k}{n}\left(Y_{k}+1\right)\right) \mid S_{n, k}\right]\right]
$$

and we have

$$
\mathbb{E}\left[S_{n} f^{\prime}\left(S_{n}\right)\right]=\frac{1}{n} \sum_{k=1}^{n} \theta \mathbb{E}\left[f^{\prime}\left(S_{n, k}+\frac{k}{n}\left(Y_{k}+1\right)\right)\right]=\frac{\theta}{n} \sum_{k=1}^{n} \mathbb{E}\left[f^{\prime}\left(S_{n}+\frac{k}{n}\right)-f^{\prime}\left(S_{n}\right)\right]+\theta \mathbb{E}\left[f^{\prime}\left(S_{n}\right)\right] .
$$


Thus, the term in the supremum of the right-hand side of (19) becomes

$$
\begin{aligned}
& \left|\frac{\theta}{n} \sum_{k=1}^{n}\left(\mathbb{E}\left[f^{\prime}\left(S_{n}\right)\right]-f^{\prime}\left(S_{n}+\frac{k}{n}\right)\right)+\theta \mathbb{E}\left[\int_{0}^{1}\left(f^{\prime}\left(S_{n}+u\right)-f^{\prime}\left(S_{n}\right)\right) d u\right]\right| \\
& \quad=\theta\left|\mathbb{E}\left[\int_{0}^{1} f^{\prime}\left(S_{n}+u\right) d u\right]-\frac{1}{n} \sum_{k=1}^{n} \mathbb{E}\left[f^{\prime}\left(S_{n}+\frac{k}{n}\right)\right]\right| \\
& \quad=\theta\left|\mathbb{E}\left[f^{\prime}\left(S_{n}+U\right)\right]-\mathbb{E}\left[f^{\prime}\left(S_{n}+\frac{I}{n}\right)\right]\right|
\end{aligned}
$$

where $U$ is a random variable uniformly distributed on $[0,1]$ and $I$ is uniformly distributed on $\{1, \ldots, n\}$ and both random variables are independent of the sequence $\left(Y_{k}\right)_{k \geq 1}$. Now, since $f$ is twice continuously differentiable on $\mathbb{R}$ with $\left\|f^{\prime}\right\|_{\infty} \leq 1$ and $\left\|f^{\prime \prime}\right\|_{\infty} \leq 1 / 2$, one gets

$$
d_{W_{2}}\left(S_{n}, X_{\theta}\right) \leq \frac{\theta}{2} \mathbb{E}\left[\left|U-\frac{I}{n}\right|\right] .
$$

To conclude the proof of Theorem 1.1, we couple $U$ and $I$ by taking $I=\lfloor n U\rfloor+1$ from which derives $\mathbb{E}\left[\left|U-\frac{I}{n}\right|\right]=1 /(2 n)$.

\subsection{Stein-Malliavin bounds for Poissonian stabilizing functionals}

In this section, we use the Stein bound (9) to present recent breakthroughs from [81] establishing likely optimal speed of convergence in CLT for some stochastic geometric functionals, see Theorem 1.4. For that purpose, the right-hand side of (9) is assessed using Malliavin calculus for Poissonian functionals, see Section 1.3.1 and the bound (22), see also [92] for such a survey of the combination Stein-Malliavin on the Gaussian space. This bound is next explicited using the notion of stabilization together with sharp variance bounds taken from the literature $[95,104]$.

Let us specify the setting: let $\mathrm{d}$ be a semi-metric on a space $\mathbb{X}$, endowed with the Borel $\sigma$-algebra $\mathscr{B}(\mathbb{X})$ and equipped with some measure $\mathbb{Q}$. We denote by $\mathscr{N}$ the set of all simple counting measures on $\mathbb{X}$. For $s \geq 1$, let $\eta_{s}$ be the Poisson point process on $\mathbb{X}$ with intensity measure $s \mathbb{Q}$. In this section, we shall give quantitative convergence bound for CLT for the following square-integrable Poissonian functionals

$$
F\left(\eta_{s}\right)=\sum_{x \in \eta_{s}} \xi\left(x, \eta_{s}\right)
$$

where the so-called score function $\xi: \mathbb{X} \times \mathscr{N} \rightarrow \mathbb{R}$ gives the contribution $\xi\left(x, \eta_{s}\right)$ of each point $x$ of the Poissonian configuration $\eta_{s}$ to the whole functional $F\left(\eta_{s}\right)$. We give below conditions under which $F\left(\eta_{s}\right)$ is asymptotically normal as $s \rightarrow+\infty$, with rates of convergence to the (standard) Gaussian distribution which are presumably optimal in two important examples: nearest neighbour statistics (Section 1.3.2), and statistics of random convex hulls (Section 1.3.3). In the sequel, we shall use the abuse of notation $F_{s}=F\left(\eta_{s}\right)$ and the standardized random variable $\widetilde{F}\left(\eta_{s}\right)=\widetilde{F}_{s}:=\left(F_{s}-\mathbb{E}\left[F_{s}\right]\right) / \operatorname{Var}\left(F_{s}\right)^{1 / 2}$.

\subsubsection{Malliavin calculus and second order Poincaré inequality}

We present some tools in Malliavin calculus to deal with the Stein bound (9). For this section, we set $s=1$ and denote $\eta:=\eta_{1}, F:=F_{1}$. The Malliavin derivative of $F$ on input $\eta$ is defined by the difference operator

$$
\mathrm{D}_{x} F(\eta)=F(\eta \cup\{x\})-F(\eta), \quad x \in \mathbb{X} .
$$


Higher order derivatives are defined by iterating this operator, i.e. for $x, y \in \mathbb{X}$, we set

$$
\mathrm{D}_{x, y}^{2} F(\eta)=\mathrm{D}_{x}\left(\mathrm{D}_{y} F\right)(\eta)=F(\eta \cup\{x, y\})-F(\eta \cup\{x\})-F(\eta \cup\{y\})+F(\eta) .
$$

The quantity $\mathrm{D}_{x, y}^{2} F(\eta)$ measures how much the contribution of $y$ is affected by the presence or absence of $x$. Since it is symmetric in $x$ and $y$, it can be seen as a measure of the interaction between $x$ and $y$. We will see that whenever $\mathrm{D}_{x, y}^{2} F$ is small for far away enough $x, y$, the contributions of distant points to $F\left(\eta_{s}\right)$ have a weak dependence, and in turn $\widetilde{F}_{s}$ becomes asymptotically normal when $s \rightarrow \infty$.

In addition to the Malliavin derivative, we consider the operator I defined by the integration by parts formula

$$
\mathbb{E}[F G]=\mathbb{E}\left[\langle\mathrm{D} G, \mid F\rangle_{L^{2}(\mathbb{Q})}\right], \quad F, G \in L^{2}(\eta)
$$

Plugging (21) with $G=f(F)$ in the Stein bound (9), its right-hand side becomes

$$
\left|\mathbb{E}\left[f^{\prime}(F)-\langle\mathrm{D} f(F), \mid F\rangle_{L^{2}(\mathbb{Q})}\right]\right|
$$

Using a Taylor expansion of $\mathrm{D} f(F)$, Peccati, Solé, Utzet, Taqqu obtain the following Stein-Malliavin bound which appear to be a brickstone in Stein's method for stochastic geometry:

Theorem 1.2 (Th. 3.1 in [91]). Let $\mathscr{D}$ be the class of centered functionals such that $\mathbb{E}\left[\mathrm{D}_{x} F^{2}\right]<+\infty$ for $\mathbb{Q}$-almost every $x \in \mathbb{X}$. Then for $F \in \mathscr{D}$, we have

$$
d_{W}(F, N) \leq \sqrt{\mathbb{E}\left[\left(1-\langle\mathrm{D} F, \mid F\rangle_{L^{2}(\mathbb{Q})}\right)^{2}\right]}+\int_{\mathbb{X}} \mathbb{E}\left[\left|\mathrm{D}_{x} F\right|^{2}\left|\mathrm{I}_{x} F\right|\right] \mathbb{Q}(d x) .
$$

Note that the operator I enjoys also a representation using the notion of thinning: for $u \in[0,1]$, attach to the points of $\eta$ independent Bernoulli random variables $B_{x}, x \in \eta$, with parameter $u$, and let $\eta_{u}=\left\{x \in \eta: B_{u}=0\right\}$. In other words, $\eta_{u}$ is obtained from $\eta$ by independently removing the points of $\eta$ with probability $u, \eta_{u}$ is called a $(1-u)$-thinning of $\eta$. It allows in particular to couple all the $\eta_{u}, u \geq 0$, on the same probability space. Then, let $\left(\eta_{u}^{\prime}\right)_{u \geq 0}$ be an independent copy of $\left(\eta_{u}\right)_{u \geq 0}$ and define the semi-group

$$
\mathrm{P}_{u} F(\eta)=\mathbb{E}\left[F\left(\eta_{u} \cup \eta_{1-u}^{\prime}\right) \mid \eta\right], \quad u \in[0,1]
$$

The operator I and its derivative admit the dynamic representation

$$
\mathrm{I} F=\int_{0}^{1} \mathrm{P}_{u} \mathrm{D} F d u \quad \text { and } \quad \mathrm{D}(\mathrm{I} F)=\int_{0}^{1} u \mathrm{P}_{u} \mathrm{D}^{2} F d u
$$

These formulae, exploited in [82] (see Corollary 3.3 therein), come from a Mehler formula (named in analogy with the Gaussian setting), as established by Privault in [102]. Another crucial tool in the study of square integrable Poissonian functionals based on Malliavin calculus is their Wiener-Itô decomposition: it is a $L^{2}$ orthogonal decomposition as a possibly infinite sum of multiple stochastic integrals with respect to the compensated Poisson measure $\eta-\mathbb{Q}$, but we do not detail it here for the sake of brevity and refer to [91] or [102] for an introduction.

Using (23) in combination with (22) in Theorem 1.2, Last, Peccati and Schulte obtain in [82, Th. 1.1] the following second order Poincaré inequality (according to the terminology introduced in [25]):

$$
d_{W}(\widetilde{F}(\eta), N) \leq \gamma_{1}+\gamma_{2}+\gamma_{3}
$$

where $\gamma_{1}, \gamma_{2}, \gamma_{3}$ only involve the moments of $\mathrm{D} \widetilde{F}$ and $\mathrm{D}^{2} \widetilde{F}$ up to the order 4 :

$$
\gamma_{1}=2\left(\int_{\mathbb{X}^{3}}\left(\mathbb{E}\left[\left(\mathrm{D}_{x_{1}} \widetilde{F}\right)^{2}\left(\mathrm{D}_{x_{2}} \widetilde{F}\right)^{2}\right]\right)^{1 / 2}\left(\mathbb{E}\left[\left(\mathrm{D}_{x_{1}, x_{3}}^{2} \widetilde{F}\right)^{2}\left(\mathrm{D}_{x_{2}, x_{3}} \widetilde{F}\right)^{2}\right]\right)^{1 / 2} \mathbb{Q}\left(d x_{1}\right) \mathbb{Q}\left(d x_{2}\right) \mathbb{Q}\left(d x_{3}\right)\right)^{1 / 2}
$$




$$
\begin{aligned}
\gamma_{2} & =\left(\int_{\mathbb{X}^{3}} \mathbb{E}\left[\left(\mathrm{D}_{x_{1}, x_{3}}^{2} \widetilde{F}\right)^{2}\left(\mathrm{D}_{x_{2}, x_{3}}^{2} \widetilde{F}\right)^{2}\right] \mathbb{Q}\left(d x_{1}\right) \mathbb{Q}\left(d x_{2}\right) \mathbb{Q}\left(d x_{3}\right)\right)^{1 / 2} \\
\gamma_{3} & =\int_{\mathbb{X}} \mathbb{E}\left[\left|\mathrm{D}_{x} \widetilde{F}\right|^{3}\right] \mathbb{Q}(d x) .
\end{aligned}
$$

As anticipated above, the bound (24) ensures that the Wasserstein distance between $\widetilde{F}(\eta)$ and $N$ is small if for distant points $x, y \in \mathbb{X}, \mathbb{E}\left[\left(\mathrm{D}_{x, y}^{2} \widetilde{F}\right)^{4}\right]$ is small. In order to explicit further this bound (24) we shall now exploit geometrical properties of the Poissonian functional (20) under study with the notion of stabilization.

\subsubsection{Stabilization}

We assume now that the metric space $(\mathbb{X}, d)$ satisfies the following property: there are constants $\kappa, \gamma>0$ such that

$$
\limsup _{\varepsilon \rightarrow 0} \frac{\mathbb{Q}\left(B_{\mathrm{d}}(x, r+\varepsilon)\right)-\mathbb{Q}\left(B_{\mathrm{d}}(x, r)\right)}{\varepsilon} \leq \kappa r^{\gamma-1}, \quad r \geq 0, x \in \mathbb{X}
$$

where $B_{\mathrm{d}}(x, r)$ is the closed ball with center $x$ and radius $r$ in the metric d. For instance, the condition (25) holds with $\gamma=d$ if $\mathbb{X}$ is a subset of $\mathbb{R}^{d}$ and $\mathbb{Q}$ has a bounded density with respect to the Lebesgue measure, or with $\gamma=m$ if $\mathbb{X}$ is a $m$-dimensional manifold with $\mathbb{Q}$ having a bounded density with respect to the $m$-dimensional Hausdorff measure $\mathcal{H}^{m}$, and $\mathrm{d}$-spheres are not too large (see [81] for details). Observe that when $\gamma$ is sharp in (25), the typical distance between neighbours in $\eta_{s}$ is of order $s^{-1 / \gamma}$, as a consequence it is relevant to consider also the scaled distance $\mathrm{d}_{s}=s^{1 / \gamma} \mathrm{d}$.

Definition 1.1. A measurable function $R: \mathbb{X} \times \mathscr{N} \rightarrow \mathbb{R}_{+}$is a radius of stabilization for the score function $\xi$ if the contribution $\xi\left(x, \eta_{s}\right)$ of a point $x \in \mathbb{X}$ to the random variable $F\left(\eta_{s} \cup\{x\}\right)$ essentially depends on the points of $\eta_{s}$ falling inside $B_{\mathrm{d}}\left(x, R\left(x, \eta_{s}\right)\right)$ : for all $\zeta \subset \mathbb{X}$ with $\# \zeta \leq 7$ (where $\# A$ stands for the cardinal of a set $A$ ),

$$
\xi\left(x, \eta_{s} \cup \zeta\right)=\xi\left(x,\left(\eta_{s} \cup \zeta\right) \cap B_{\mathrm{d}}\left(x, R\left(x, \eta_{s}\right)\right)\right), \quad x \in \mathbb{X}, s \geq 1 .
$$

In this case, the score $\xi$ is said to be stabilizing.

The presence of $\zeta$ with $\# \zeta \leq 7$ in Def. 1.1 is a technical requirement that does not affect much the class of stabilizing functionals. Let us give a classical example on $\mathbb{X}=[0,1]^{d}$, endowed with the Euclidean metric $\mathrm{d}^{e}$. Let $\xi\left(x, \eta_{s}\right)$ be the distance from $x$ to its nearest neighbour in $\eta_{s} \backslash\{x\}$ (i.e. the $\mathrm{d}^{e}$-closest element of $x$ in $\left.\eta_{s} \backslash\{x\}\right)$ so that $F\left(\eta_{s}\right)$ in (20) is the total length of the oriented nearest neighbour graph. Then it is immediate that $R\left(x, \eta_{s}\right):=\mathrm{d}^{e}\left(x, \eta_{s} \backslash x\right)$ is a stabilization radius since $\eta_{s} \cap B_{\mathrm{d}^{e}\left(x, R\left(x, \eta_{s}\right)\right)}$ is reduced to the set consisting of $x$ and its nearest neighbour(s) in $\eta_{s} \backslash\{x\}$. Moreover since $\eta_{s}$ is stationary, we have

$$
\mathbb{P}\left(s^{1 / \gamma} R\left(x, \eta_{s}\right) \geq r\right)=\mathbb{P}\left(\eta_{s} \cap B_{\mathrm{d}_{s}^{e}}(0, r)=\emptyset\right)=\exp \left(-\kappa_{d} r^{d}\right), \quad x \in \mathbb{X}, s \geq 1
$$

where $\kappa_{d}$ is the Lebesgue measure of the Euclidean unit ball of $\mathbb{R}^{d}$ so that the following property is satisfied for the score given by the distance to the nearest neighbour:

Definition 1.2. A score function $\xi$ is said to be exponentially stabilizing if for some $C, c, \alpha>0$,

$$
\mathbb{P}\left(s^{1 / \gamma} R\left(x, \eta_{s}\right) \geq r\right) \leq C \exp \left(-c r^{\alpha}\right), \quad x \in \mathbb{X}, s>1, r \geq 0 .
$$

Such exponential decay as in (26) ensures that the contributions of distant points to $F\left(\eta_{s}\right)$ in $(20)$ are essentially independent. The interest of the radii of stabilization to minimize Stein bound as in (24) comes from the following result (Lemma 5.3 in [81]):

Proposition 1.1. Let $\xi$ be a score stabilized by a radius $R$. For $x, y_{1}, y_{2} \in \mathbb{X}$ such that $R(x, \eta)<\left\|x-y_{i}\right\|$ for $i=1,2$, we have $\mathrm{D}_{y_{1}, y_{2}}^{2} \xi(x, \eta)=0$. 
As a consequence, by linearity of $\mathrm{D}^{2}$, Proposition 1.1 ensures that $\mathrm{D}_{x, y}^{2} F$ is expected to be small for distant $x, y$ when the scores are stabilizing, and hence in virtue of $(24), F\left(\eta_{s}\right)$ is expected to be close to the Gaussian distribution. For that very purpose, an additional parameter can be used: let $A \subseteq \mathbb{X}$ be a set for which the score of points of $\eta_{s}$ falling far away from $A$ have a large chance to vanish, i.e. for some $\beta, C>0, c \geq 0, \zeta \subset \mathbb{X}$ with $\# \zeta \leq 7$

$$
\mathbb{P}\left(\xi\left(x, \eta_{s} \cup \zeta\right) \neq 0\right) \leq C \exp \left(-c \mathrm{~d}_{s}(x, A)^{\beta}\right), \quad x \in \mathbb{X}, s \geq 1 .
$$

In the example of nearest neighbours, the choice $A=\mathbb{X}=[0,1]^{d}, C=1, c=0$ trivially fits this definition and $I_{s}$ below in Theorem 1.3 is just equal to $s$. Actually, the introduction of $A$ is interesting if its dimension is smaller than the ambient dimension. In Section 1.3.3, we give an example where $A$ is a $(d-1)$-dimensional submanifold of $\mathbb{R}^{d}$ but first we give a Wasserstein bound from [81] for a CLT for $F_{s}=F\left(\eta_{s}\right)$ with exponentially stabilizing scores enjoying this extra condition (27):

Theorem 1.3 (Theorem 2.1.a in [81]). Assume that the score functions $\xi$ are exponentially stabilizing, (27) is satisfied for a set $A$ and the moment condition

$$
\sup _{s \geq 1, x \in \mathbb{X},|\zeta| \leq 7} \mathbb{E}\left[\# \xi\left(x, \eta_{s} \cup \zeta\right)\right]^{4+p}<+\infty .
$$

Let $I_{s}:=s \int_{\mathbb{X}} \exp \left(-c_{0} \mathrm{~d}_{s}(x, A)^{\beta}\right) \mathbb{Q}(d x)$ with small enough $c_{0}$. Then

$$
d_{W}\left(\frac{F_{s}-\mathbb{E}\left[F_{s}\right]}{\sqrt{\operatorname{Var}\left(F_{s}\right)}}, N\right) \leq C^{\prime}\left(\frac{\sqrt{I_{s}}}{\operatorname{Var}\left(F_{s}\right)}+\frac{I_{s}}{\operatorname{Var}\left(F_{s}\right)^{3 / 2}}+\frac{I_{s}^{5 / 4}+I_{s}^{3 / 2}}{\operatorname{Var}\left(F_{s}\right)^{2}}\right) .
$$

Note that, using different tools, a very similar result can also be established for binomial input instead of Poissonian input, i.e. samples of $n$ iid points, see [81, Th. 2.1.b]. Theorem 1.3 applies to some nearest neighbour statistics and recovers presumably optimal convergence rate in $s^{-1 / 2}$ to the (standard) normal distribution when we use sharp variance estimates from [104], see [81, Th. 3.1].

\subsubsection{Random convex hulls}

In this section, we present the application of Theorem 1.3 to an old problem from stochastic geometry, and show how Theorem 1.3 improves pre-existing bounds in most situations, we refer again to [81, Section 3.4] for details. Assume that $\mathbb{X}$ is a compact convex subset of $\mathbb{R}^{d}, d \geq 2$, with a smooth $\mathcal{C}^{2}$ boundary having everywhere positive Gaussian curvature, and let $\mathbb{Q}$ be Lebesgue measure restricted to $\mathbb{X}$. Let $\mathcal{C}_{s}$ be the convex hull of the Poisson point process $\eta_{s}$. We aim to obtain the limit behaviour of a real functional $V\left(\mathcal{C}_{s}\right)$ where $V$ is any of the $d$ intrinsic volumes $V_{i}$ of $\mathbb{R}^{d}, i=1, \ldots, d$. Recall that $V_{d}$ is the volume, and $V_{d-1}$ is the $(d-1)$-dimensional Hausdorff measure of the boundary. The functional $V$ can also be taken to be the number $f_{k}$ of $k$-simplexes of points on the boundary of a convex body. For instance $f_{0}\left(\mathcal{C}_{s}\right)=\# \operatorname{ext}\left(\mathcal{C}_{s}\right)$ is the number of extremal points of $\eta_{s}, f_{1}\left(\mathcal{C}_{s}\right)$ is the number of edges, etc. All these functionals admit a local score representation of the form (20). For instance, $V_{d-1}\left(\mathcal{C}_{s}\right)=\sum_{x \in \eta_{s}} \xi\left(x, \eta_{s}\right)$ and $f_{k}\left(\mathcal{C}_{s}\right)=\sum_{x \in \eta_{s}} \xi_{k}\left(x, \eta_{s}\right)$ with

$$
\begin{aligned}
\xi\left(x, \eta_{s}\right) & =\mathbf{1}_{\left\{x \in \operatorname{ext}\left(\mathcal{C}_{s}\right)\right\}} \mathcal{H}^{d-1}\left(\left\{y \in \partial \mathcal{C}_{s}:\|y-x\| \leq \mathrm{d}^{e}\left(y, \eta_{s} \cap \partial \mathcal{C}_{s} \backslash\{x\}\right)\right\}\right), \\
\xi_{k}\left(x, \eta_{s}\right) & =\mathbf{1}_{\left\{x \in \operatorname{ext}\left(\mathcal{C}_{s}\right)\right\}} \frac{1}{k+1} \#\left\{k \text {-simplexes of } \partial \mathcal{C}_{s} \cap \eta_{s} \text { containing } x\right\} .
\end{aligned}
$$

In this situation, Theorem 1.3 specifies as follows, see [81, Th. 5.5]:

Theorem 1.4. Let $V \in\left\{V_{1}, \ldots, V_{d}, f_{0}, \ldots, f_{d-1}\right\}$ and consider $F\left(\eta_{s}\right)=V\left(\mathcal{C}_{s}\right)$. Then

$$
d_{W}\left(\frac{F_{s}-\mathbb{E}\left[F_{s}\right]}{\sqrt{\operatorname{Var}\left(F_{s}\right)}}, N\right) \leq C s^{-\frac{d-1}{2(d+1)}} .
$$


Let us indicate the general line of the proof. First, the set whose neighbourhood is relevant is $A=\partial \mathbb{X}$, in the sense that points far away from $A$ have a large chance to give a zero contribution, in view of (27). The second important point is the choice of the metric $\mathrm{d}$ :

$$
\mathrm{d}(x, y)=\max \left(\|x-y\|, \sqrt{\left|\mathrm{d}_{e}(x, A)-\mathrm{d}_{e}(y, A)\right|}\right), \quad x, y \in \mathbb{X}
$$

For $x$ far away from $A$, a ball of the form $B_{\mathrm{d}}(x, r)$ is comparable to $B_{\mathrm{d}^{e}}(x, r)$, while for $x$ getting close to $A$ the ball $B_{\mathrm{d}}(x, r)$ becomes more and more flat with the small axis orthogonal to the boundary surface. This geometry is indeed adapted to the dependency of the problem since if $x$ is an extremal point, only the other extremal points, hence falling close to $A$ and being in $B_{\mathrm{d}}(x, r)$, can have an influence on the contribution of $x$. We can show that $\mathrm{d}$ satisfies (25) with $\gamma=d$, and the rest of the proof consists in showing moment conditions and exponential stabilization with respect to the metric d. A good deal of integral geometry is involved in this kind of proof together with variance estimates for convex hull functionals taken from the literature, see for instance the breakthrough paper of Reitzner [104] and references therein.

\section{FunCtional Limit THEOREMS FOR CORRELATED RANDOM WALKS AND RANDOM FIELDS}

In this section, we are interested in functional limit theorems generalizing Donsker's invariance principle (6) under dependence condition and/or for random fields. Recall that for iid random variables $\left(X_{i}\right)_{i \geq 1}$ with, say, zero mean and unit variance, the associated piecewise constant random walk $\left(S_{\lfloor n t\rfloor}\right)_{t \in[0,1]}$ satisfies

$$
\left(\frac{S_{\lfloor n t\rfloor}}{\sqrt{n}}\right)_{t \in[0,1]} \stackrel{J_{1}}{\Longrightarrow}\left(B_{t}\right)_{t \in[0,1]} \quad \text { in } \mathbb{D}([0,1])
$$

when $n \rightarrow+\infty$ where $\left(B_{t}\right)_{t \in[0,1]}$ is a standard Brownian motion. When the $X_{i}, i \geq 1$, have Rademacher distribution $\frac{1}{2} \delta_{-1}+\frac{1}{2} \delta_{1}$, this result justifies that the simple random walk can be seen as a simple discrete counterpart of the Brownian motion. Generalizations of (28) are well known for square integrable stationary random variables $\left(X_{i}\right)_{i \geq 1}$ under weak dependence including summable mixing coefficients, martingale and martingale approximations, etc. See $[18,37,87]$ and references therein. The common feature in all these settings is that the variance $\operatorname{Var}\left(S_{n}\right)$ is asymptotically of order $n$ (up to a possibly slowly varying function factor, see [87]) and the limit process is always a Brownian motion. This is due to the fact that the Brownian motion is the only (1/2)-self-similar Gaussian process with stationary increments.

The picture becomes different when the dependence structure in the stationary sequence $\left(X_{i}\right)_{i \geq 1}$ becomes stronger. Several notions of strong dependence have been considered in the literature (long memory, long-range dependence, etc) but there is no universal definition, see [101,110]. Typically, under strong dependence, if $\operatorname{Var}\left(S_{n}\right)$ still grows to infinity, it is at a different rate than in the iid case. In general, $\operatorname{Var}\left(S_{n}\right)=n^{2 H} \ell(n)$ with $H \in[0,1]$ and $\ell$ a slowly varying function, and the $\left(X_{i}\right)_{i \geq 1}$ are said to be strongly dependent if $H \neq 1 / 2$. The notion of long-range dependence usually corresponds to the case $H>1 / 2$.

The fractional Brownian motion $B^{(H)}=\left(B_{t}^{(H)}\right)_{t \in[0,1]}$ with Hurst parameter $H \in(0,1)$ is the centered Gaussian process with covariances

$$
\operatorname{Cov}\left(B_{t}^{(H)}, B_{s}^{(H)}\right)=\frac{1}{2}\left(t^{2 H}+s^{2 H}-|t-s|^{2 H}\right), \quad t, s>0
$$

Up to a multiplicative constant, it is the only Gaussian process that is $H$-self-similar and has stationary increments, see e.g. [111]. As a consequence, when invariance principles for partial sums of stationary random variables can be established, the limit processes are necessarily fractional Brownian motions. Such an invariance principle was obtained by Davydov [34] in the setting of linear processes with independent innovations and it has 
been extended by several authors [38,117]. See also the book [101] for more limit theorems involving fractional Brownian motions.

A natural question is then the following: can one find a simple dependence structure that allows to build a random walk (necessarily with correlated steps) that can be seen as a discrete counterpart of the fractional Brownian motion of parameter $H \neq 1 / 2$, like the simple random walk for the Brownian motion?

Such a construction from [10] is discussed in the following Section 2.1 before considering random field version of such question in Section 2.2.

\subsection{Correlated random walks}

In 2013, Hammond and Sheffield propose in [71] a model of correlated random walk $S_{n}=X_{1}+\cdots+X_{n}$, $n \geq 1$, that, properly scaled, converge to a fractional Brownian motion $B^{(H)}$ with Hurst parameter $H>1 / 2$, see Theorem 2.1 below. The correlation between the steps $X_{i}, i \geq 1$, of the walk $S_{n}$ is created via a random partition of the set of their indices $\mathbb{N}^{*}=\{1,2, \ldots\}$ which is itself the trace of a random partition $\Pi_{\mu}$ of $\mathbb{Z}$.

Let $\left(J_{i}\right)_{i \in \mathbb{Z}}$ be a family of iid random variables with common distribution $\mu$ on $\mathbb{N}^{*}$; consider the random graph with $\mathbb{Z}$ as set of vertices and having, for each $i \in \mathbb{Z}$, an oriented edge from $i$ to $i-J_{i}$. The random partition $\Pi_{\mu}$ of $\mathbb{Z}$ considered is then given by the connected components of this random graph. To describe this partition for all $i \in \mathbb{Z}$, set $A_{i}$ for the ancestral line of $i$, i.e. the infinite set obtained, starting from $i$, by following the oriented edges: $A_{i}=\left\{i, i-J_{i}, i-J_{i}-J_{i-J_{i}}, \ldots\right\}$. We say that two indices $i$ and $j \in \mathbb{Z}$ are in the same component of the random partition (denoted by $i \sim_{\Pi_{\mu}} j$ ) if and only if $A_{i} \cap A_{j} \neq \emptyset$. The random variables $\left(X_{i}\right)_{i \in \mathbb{Z}}$ are then taken identically distributed with Rademacher distribution with $X_{i}=X_{j}$ whenever $i \sim_{\Pi_{\mu}} j$ and $X_{i}$ independent of $X_{j}$ otherwise.

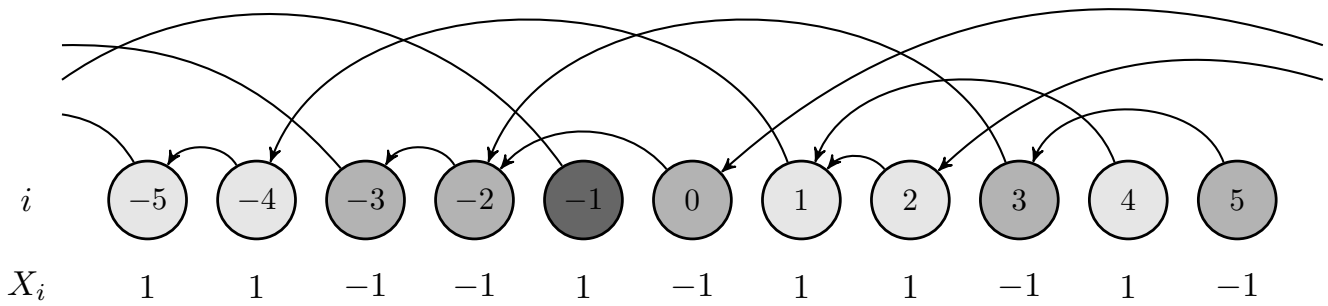

FiguRE 1. Illustration of the ancestral lines and related correlated steps $X_{i}$ 's.

The measure $\mu$ on $\mathbb{N}$ is assumed to have a regular varying tail, that is $\mu([n,+\infty))=n^{-\alpha} \ell(n)$ for $\alpha \in(0,1)$ and $\ell$ a slowly varying function. As a consequence, $\mu$ belongs to the domain of attraction of a totally skewed $\alpha$-stable random variable, see [111].

Hammond and Sheffield prove in [71] that the random partition $\Pi_{\mu}$ has almost surely only one component when $\alpha>1 / 2$ (actually also when $\alpha=1 / 2$ if $\ell$ is constant, see [10]) and has infinitely many components when $\alpha<1 / 2$. In the sequel, we thus assume $\alpha \in(0,1 / 2)$. Introducing the filtration $\mathcal{F}_{j}=\sigma\left(X_{k}: k<j\right), j \in \mathbb{Z}$, one can prove that

$$
X_{k}=\sum_{j \in \mathbb{Z}}\left(\mathbb{E}\left[X_{k} \mid \mathcal{F}_{j+1}\right]-\mathbb{E}\left[X_{k} \mid \mathcal{F}_{j}\right]\right)=: \sum_{j \in \mathbb{Z}} \Delta_{j}\left(X_{k}\right) .
$$

The sequence $\left(X_{j}^{*}\right)_{j \in \mathbb{Z}}:=\left(\Delta_{j}\left(X_{j}\right)\right)_{j \in \mathbb{Z}}$ is a sequence of martingale differences with respect to the filtration

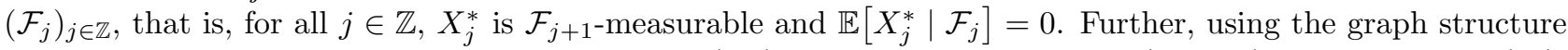
of the model, one can show that for all $j<k, \Delta_{j}\left(X_{k}\right)=q_{k-j} X_{j}^{*}$, where $q_{n}=\mathbb{P}\left(0 \in A_{n}\right)$. Together with (30), this leads to a representation of the sequence $\left(X_{k}\right)_{k \in \mathbb{Z}}$ as a linear process with martingale-difference innovations:

$$
X_{k}=\sum_{j \in \mathbb{Z}} q_{k-j} X_{j}^{*}, \quad k \in \mathbb{Z} .
$$


From this representation, one can obtain an invariance principle (see e.g. the central limit theorem for linear process with martingale-difference innovations in [10]). It is also a consequence of a stronger result in [71] that uses a similar approach based on martingale approximations and states as follows:

Theorem 2.1 (Th.1.1 in [71]). For the Hammond-Sheffield model described above with $\alpha \in(0,1 / 2)$, we have

$$
\left(\frac{S_{\lfloor n t\rfloor}}{n^{\frac{1}{2}+\alpha} \ell(n)^{-1}}\right)_{t \in[0,1]} \stackrel{J_{1}}{\Longrightarrow} \sigma\left(B_{t}^{\left(\frac{1}{2}+\alpha\right)}\right)_{t \in[0,1]} \quad \text { in } \mathbb{D}([0,1]) \text {, as } n \rightarrow+\infty
$$

where

$$
\sigma^{2}=\left(2 \alpha(2 \alpha+1) \Gamma(1-\alpha)^{2} \Gamma(2 \alpha) \cos (\alpha \pi) \sum_{k \geq 0} q_{k}^{2}\right)^{-1} .
$$

The order of normalization $n^{\frac{1}{2}+\alpha}$ in Theorem 2.1 comes from the regular variation of the measure $\mu$ which implies $1-\varphi_{\mu}(t) \sim t^{\alpha} \ell\left(t^{-1}\right)$ for the characteristic function of $\mu$ in the neighbourhood of 0 and relates as follows to $\operatorname{Var}\left(S_{n}\right)$ : using successively the expression $S_{n}=\sum_{j \in \mathbb{Z}} b_{n, j} X_{j}^{*}$ in terms of $b_{n, j}=\sum_{k=1}^{n} q_{k-j}$, the Parseval identity relating the $b_{n, j}$ to the Fourier series $Q$ with coefficients $q_{k}$, we have, up to multiplicative constant,

$$
\operatorname{Var}\left(S_{n}\right)=\sum_{j \in \mathbb{Z}} b_{n, j}^{2} \approx \int_{-\pi}^{\pi}|Q(x)|^{2} D_{n}(x) \overline{D_{n}(x)} d x
$$

where $D_{n}$ is a Dirichlet kernel. Finally, $\operatorname{Var}\left(S_{n}\right) \approx n^{1+2 \alpha} \ell(n)^{-2}$ comes from a simple change of variable in the latter integral and the relation $Q=\left(1-\varphi_{\mu}\right)^{-1}$ deriving from $q_{0}=1$ and for $k \geq 1$, since each edge in the graph is generated independently at each site,

$$
q_{k}=\sum_{j \geq 1} \mathbb{P}\left(0 \in A_{k}, J_{k}=j\right)=\sum_{j \geq 1} \mathbb{P}\left(0 \in A_{k-j}\right) \mathbb{P}\left(J_{k}=j\right)=\sum_{j \geq 1} \mu(j) q_{k-j} .
$$

Remark 2.1. A similar model is proposed in [46] for the case $H<1 / 2$. It is based on a simpler random partition of the set of indices $\mathbb{N}^{*}$ that uses an infinite urn scheme studied by Karlin in [76]. This random partition has infinitely many components and is obtained by choosing at random the component associated to each index in $\mathbb{N}^{*}$, independently from one index to another. Once the partition is sampled, in order to obtain negative correlation, the steps values are taken by alternating between 1 and -1 inside each component. The choice between $1,-1,1, \ldots$ or $-1,1,-1, \ldots$ is done with equal probability and independently from one component to another. See [46, Section 2.3] for more details.

Remark 2.2. Before that, Enriquez proposed in 2004 in [53] another model of simple correlated random walks where the steps $X_{i}$ 's are defined using a persistence parameter $p \in(0,1)$. Let $\left(\theta_{i}\right)_{i \geq 1}$ be iid random variables with geometric distribution of parameter $p$ and let $\Theta_{i}=\theta_{1}+\cdots+\theta_{i}, i \geq 1$. One first choose at random the value of $X_{1}$ (with Rademacher distribution) and then $X_{2}=\cdots=X_{\Theta_{1}}=X_{1}, X_{\Theta_{1}+1}=\cdots=X_{\Theta_{2}}=-X_{1}$, $X_{\Theta_{2}+1}=\cdots=X_{\Theta_{3}}=X_{1}, \ldots$ If $p=1 / 2$, it is just the simple random walk, but here extra randomness is introduced with the persistence $p$. For $H>1 / 2$, it is assumed that $p$ follows the distribution on $(1 / 2,1)$ with density $x \mapsto(1-H) 2^{3-2 H}(1-x)^{1-2 H} \mathbf{1}_{(1 / 2,1)}(x)$. The main difference with the previous correlated model is that, to obtain the desired result, aggregation is required. One has to consider an average of a large number of independent such random walks (with the persistence sampled for each walk). The invariance principles are thus more complex as they involve a double limit. A random-field version of this model is also investigated in [113].

\subsection{Correlated Random fields}

We now turn to the random field setting. All the results of this section hold in any dimension but to simplify the presentation, we only consider the case of dimension 2 . In the random field setting, one cannot talk about 
random walks any longer. For a stationary field of real random variables $\left(X_{\boldsymbol{i}}\right)_{\boldsymbol{i} \in \mathbb{N}^{2}}$, the partial sum $S_{\boldsymbol{n}}$ is defined by summing over the rectangle $R_{\boldsymbol{n}}=\left(\left[1, n_{1}\right] \times\left[1, n_{2}\right]\right) \cap\left(\mathbb{N}^{*}\right)^{2}$, where $\boldsymbol{n}=\left(n_{1}, n_{2}\right) \in\left(\mathbb{N}^{*}\right)^{2}$, that is

$$
S_{\boldsymbol{n}}=\sum_{\boldsymbol{i} \in R_{\boldsymbol{n}}} X_{\boldsymbol{i}}=\sum_{i_{1}=1}^{n_{1}} \sum_{i_{2}=1}^{n_{2}} X_{\left(i_{1}, i_{2}\right)}
$$

Observe that bold characters mean multi-indices quantities. We first consider the iid case. Let the random variables $\left(X_{\boldsymbol{i}}\right)_{\boldsymbol{i} \in \mathbb{N}^{2}}$ be $i i d$, centered, and with unit variance. Then

$$
\left(\frac{S_{\lfloor\boldsymbol{n} \cdot \boldsymbol{t}\rfloor}}{\sqrt{n_{1} n_{2}}}\right)_{\boldsymbol{t} \in[0,1]^{2}} \stackrel{J_{1}}{\Longrightarrow}\left(\mathbb{B}_{\boldsymbol{t}}\right)_{\boldsymbol{t} \in[0,1]^{2}} \quad \text { in } \mathbb{D}\left([0,1]^{2}\right), \text { as } \min \left(n_{1}, n_{2}\right) \rightarrow+\infty
$$

where $\mathbb{B}$ is the standard Brownian sheet, that is the centered Gaussian field with covariances

$$
\operatorname{Cov}\left(\mathbb{B}_{\boldsymbol{s}}, \mathbb{B}_{\boldsymbol{t}}\right)=\prod_{i=1}^{2} \operatorname{Cov}\left(B_{s_{i}}, B_{t_{i}}\right)=\prod_{i=1}^{2} \frac{1}{2}\left(s_{i}+t_{i}-\left|t_{i}-s_{i}\right|\right), \quad \boldsymbol{s}, \boldsymbol{t} \in[0,1]^{2}
$$

see [121]. Note that, as soon as both coordinates of the vector $\boldsymbol{n}$ goes to infinity, the convergence (32) holds and the limit field is always the Brownian sheet. This classical result has also been generalized for weakly dependent random fields (see e.g. [36, 52,108,120] among others) and the limit field remains the Brownian sheet.

As before in Section 2.1 for dimension one, in a strong dependence setting, new limit fields appear. As rectangular partial sums of stationary $L^{2}$-families are considered, a limit field $W$ should be Gaussian with stationary rectangular increments where such increment, say between $s$ and $\boldsymbol{t}$ are defined by $W_{\boldsymbol{t}}-W_{t_{1}, s_{2}}-$ $W_{s_{1}, t_{2}}+W_{\boldsymbol{s}}$. Further, if $\operatorname{Var}\left(S_{\boldsymbol{n}}\right)=n_{1}^{2 H_{1}} n_{2}^{2 H_{2}} \ell(\boldsymbol{n})$ (with a slowly varying function $\ell$ ), we expect a normalization of order $n_{1}^{H_{1}} n_{2}^{H_{2}} \ell(\boldsymbol{n})^{1 / 2}$ and the limit field $W$ should have an operator-self-similarity property of the form $\left(W_{\lambda^{\boldsymbol{E}} \boldsymbol{t}}\right)_{t \in \mathbb{R}^{2}} \stackrel{f d d}{=}\left(\lambda^{2} W_{\boldsymbol{t}}\right)_{t \in \mathbb{R}^{2}}$ where $\stackrel{f d d}{=}$ stands for the equality of all finite dimensional distributions and $\boldsymbol{E}$ is the diagonal matrix $\operatorname{diag}\left(1 / H_{1}, 1 / H_{2}\right)$ and $\lambda^{\boldsymbol{E}}=\operatorname{diag}\left(\lambda^{1 / H_{1}}, \lambda^{1 / H_{2}}\right)$. Natural candidates are the fractional Brownian sheets $\mathbb{B}^{(\boldsymbol{H})}, \boldsymbol{H}=\left(H_{1}, H_{2}\right) \in(0,1)^{2}$, that are the centered Gaussian random fields with covariances

$$
\operatorname{Cov}\left(\mathbb{B}_{\boldsymbol{s}}^{(\boldsymbol{H})}, \mathbb{B}_{\boldsymbol{t}}^{(\boldsymbol{H})}\right)=\prod_{i=1}^{2} \operatorname{Cov}\left(B_{s_{i}}^{\left(H_{i}\right)}, B_{t_{i}}^{\left(H_{i}\right)}\right)=\prod_{i=1}^{2} \frac{1}{2}\left(s_{i}^{2 H_{i}}+t_{i}^{2 H_{i}}-\left|t_{i}-s_{i}\right|^{2 H_{i}}\right), \quad \boldsymbol{s}, \boldsymbol{t} \in[0,1]^{2}
$$

However, an important difference with the dimension one is that the fractional Brownian sheets are not the only operator-self-similar Gaussian random fields with stationary rectangular increments. As a consequence, the class of possible limit fields is wider. We shall illustrate this idea with the two following examples that generalize the model of Section 2.1.

\subsubsection{A first generalization of Hammond-Sheffield's model}

The Hammond-Sheffield model can naturally be extended to a two-dimensional field by generating a random partition of the set of indices $\left(\mathbb{N}^{*}\right)^{2}$. To do so, consider the random graph on $\mathbb{Z}^{2}$ obtained by associating an oriented edge from $\boldsymbol{i}$ to $\boldsymbol{i}-\boldsymbol{J}_{\boldsymbol{i}}$ to each vertex $\boldsymbol{i} \in \mathbb{Z}^{2}$, the jumps $\left(\boldsymbol{J}_{\boldsymbol{i}}\right)_{\boldsymbol{i} \in \mathbb{Z}^{2}}$ being iid with common distribution $\boldsymbol{\mu}$ on $\left(\mathbb{N}^{*}\right)^{2}$. Again, the partition is taken with respect to the connected components of the graph.

The random field $\left(X_{i}\right)_{i \in \mathbb{Z}^{2}}$ is then obtained like before in Section 2.1 by taking the same random value for indices in the same component, each random value being sampled independently in each component with respect to the Rademacher distribution. Further, $\boldsymbol{\mu}$ is assumed to be in the strict domain of normal attraction of an infinitely divisible $\boldsymbol{E}$-operator stable probability $\boldsymbol{\nu}$ on $\mathbb{R}_{+}^{2}$, where $\boldsymbol{E}=\operatorname{diag}\left(1 / \alpha_{1}, 1 / \alpha_{2}\right)$, and $\alpha_{1}, \alpha_{2} \in(0,1)$. That is, if $\left(\boldsymbol{\eta}_{i}\right)_{i \geq 1}$ are iid copies with distribution $\boldsymbol{\mu}$, then $n^{-\boldsymbol{E}} \sum_{i=1}^{n} \boldsymbol{\eta}_{i} \Longrightarrow \boldsymbol{\nu}$. One typical example to keep in mind is $\boldsymbol{\mu}=\mu_{1} \otimes \mu_{2}$, where $\mu_{i}([n,+\infty)) \sim c_{i} n^{-\alpha_{i}}, i=1,2$. 


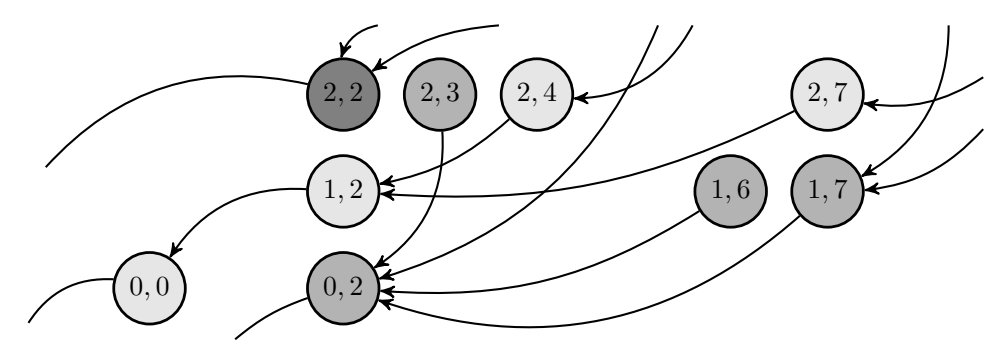

FIGURE 2. Illustration of the ancestral lines in dimension 2.

In this setting, invariance principles can be established but two important (new) facts have to be noticed. First, a scaling-transition phenomena appears: Considering rectangular partial sums, the growth rates of both directions of the rectangle $R_{n}$ (relatively to each other) has an influence on the nature of the limit field that shows up in the invariance principle (and also on the normalization term). The critical case, where the full dependence structure persists at the limit, only occurs when $\boldsymbol{n}=n^{\boldsymbol{E}} \mathbf{1}=\left(n^{1 / \alpha_{1}}, n^{1 / \alpha_{2}}\right)$. This contrasts with the iid case where the growth rate has no influence. The second fact to be noticed is that, even in the critical case, the limit random field does not have a sheet structure. The result from [10, Th. 1] states as follows:

Theorem 2.2. For $S_{n}^{\boldsymbol{E}}(\boldsymbol{t})=\sum_{i \in R_{\lfloor n} E_{t\rfloor}} X_{i}$,

$$
\left(\frac{S_{n}^{\boldsymbol{E}}(\boldsymbol{t})}{n^{1+\operatorname{tr}(\boldsymbol{E}) / 2}}\right)_{\boldsymbol{t} \in[0,1]^{2}} \stackrel{J_{1}}{\Longrightarrow} \sigma\left(\mathbb{W}_{\boldsymbol{t}}\right)_{\boldsymbol{t} \in[0,1]^{2}} \quad \text { in } \mathbb{D}\left([0,1]^{2}\right)
$$

when $n \rightarrow+\infty$ where $\mathbb{W}$ is the centered Gaussian field with covariance

$$
\operatorname{Cov}\left(\mathbb{W}_{\boldsymbol{s}}, \mathbb{W}_{\boldsymbol{t}}\right)=\int_{\mathbb{R}^{2}}\left|\log \varphi_{\boldsymbol{\nu}}(\boldsymbol{y})\right|^{-2} \prod_{r=1}^{2} \frac{\left(e^{i t_{r} y_{r}}-1\right) \overline{\left(e^{i s_{r} y_{r}}-1\right)}}{2 \pi\left|y_{r}\right|^{2}} d \boldsymbol{y},
$$

$\varphi_{\boldsymbol{\nu}}$ is the characteristic function of $\boldsymbol{\nu}$ and $\sigma^{2}=\left(\sum_{\boldsymbol{k} \in \mathbb{Z}^{2}} q_{\boldsymbol{k}}^{2}\right)^{-1}$ where $q_{\boldsymbol{k}}$ is the probability that $\mathbf{0}$ belongs to the ancestral line of the vertex $\boldsymbol{k}$ in the two-dimensional random graph of Figure 2.

Even when $\boldsymbol{\mu}=\mu_{1} \otimes \mu_{2}$ (then $\boldsymbol{\nu}$ is such that $\log \varphi_{\boldsymbol{\nu}}(\boldsymbol{y})=a_{1} y_{1}^{\alpha_{1}}+a_{2} y_{2}^{\alpha_{2}}$ for some $\left.a_{1}, a_{2} \in \mathbb{R}\right)$, the process $\mathbb{W}$ is not a fractional Brownian sheet and it seems to be new in the literature. Outside the critical case, the normalization has to be adapted and the limit random field is degenerated in one direction, exhibiting independence or complete dependence in that direction. For example, considering partial sums $S_{n}^{\boldsymbol{E}^{\prime}}(\boldsymbol{t})$ with $\boldsymbol{E}^{\prime}=\operatorname{diag}\left(1 / \alpha_{1}, 1 / \alpha_{2}^{\prime}\right)$ and $\alpha_{2}^{\prime}>\alpha_{2}$, the limit field is a degenerated fractional Brownian sheet $\mathbb{B}^{(\boldsymbol{H})}$ in the sense that $H_{1}=1 / 2$ when $\alpha_{2}<1 / 2$ or $H_{2}=1$ when $\alpha_{2}>1 / 2$. See [10, Th. 2] for the exact details. This phenomenon of having different limits depending on the rectangle's growth in the summation has been named scaling transition in a series of papers $[99,100,103]$ where the same phenomenon was observed in other models, all involving random fields with long-range dependence.

Theorem 2.2 above is proved in [10] following the approach of representing the field as a linear field with martingale-difference innovations, the martingale differences being well defined with respect to the lexicographical order $\prec_{\text {lex }}$ on $\mathbb{Z}^{2}$. This is essentially due to the fact that, thanks to the South-West orientation of the random graph, the variable $X_{\boldsymbol{j}}$ can be written as $\sum_{\boldsymbol{k} \in\left(\mathbb{N}^{*}\right)^{2}} X_{\boldsymbol{j}-\boldsymbol{k}} \mathbf{1}_{\left\{\boldsymbol{J}_{\boldsymbol{j}}=\boldsymbol{k}\right\}}$, and thus

$$
\mathbb{E}\left[X_{\boldsymbol{j}} \mid X_{\boldsymbol{i}}: i_{1}<j_{1} \text { or } i_{2}<j_{2}\right]=\mathbb{E}\left[X_{\boldsymbol{j}} \mid X_{\boldsymbol{i}}: i_{1}<j_{1} \text { and } i_{2}<j_{2}\right]=\mathbb{E}\left[X_{\boldsymbol{j}} \mid X_{\boldsymbol{i}}: \boldsymbol{i} \prec_{\text {lex }} \boldsymbol{j}\right] \text {. }
$$

This possibility of using the lexicographical order allows to turn the problem into a dimension-free problem. 


\subsubsection{Fractional Brownian sheet}

The preceding model in Section 2.2.1 shows that fractional Brownian sheets only appear in degenerated situations. However, adapting the construction of the underlying partition, other discrete models can be considered, in particular models that scale to any fractional Brownian sheets with $\boldsymbol{H} \in(1 / 2,1)^{2}$.

Considering two independent random partitions $\Pi_{\mu_{1}}$ and $\Pi_{\mu_{2}}$ of $\mathbb{Z}$ as in Section 2.1, the partition $\Pi_{\mu_{1}, \mu_{2}}$ of $\mathbb{Z}^{2}$ is then given by the Cartesian products $C_{1} \times C_{2}$, for all $C_{1} \in \Pi_{\mu_{1}}, C_{2} \in \Pi_{\mu_{2}}$ as illustrated in Figure 3 . The probability distributions $\mu_{i}, i=1,2$, are assumed to have a regular varying tail with index $\alpha_{i} \in(0,1 / 2)$ :

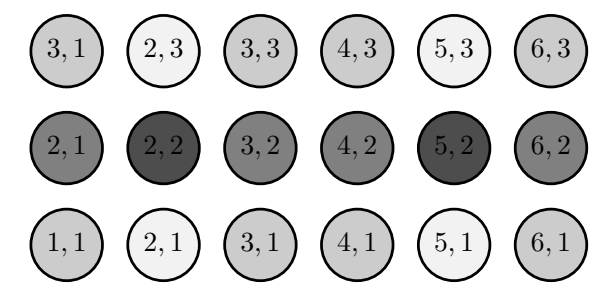

Figure 3. The partition obtained from $\{\{1,3,4,6\},\{2,5\}\}$ (horizontal) and $\{\{1,3\},\{2\}\}$ (vertical).

$\mu_{i}([n,+\infty))=n^{-\alpha_{i}} \ell_{i}(n), i=1,2$. As before, let the random field $\left(X_{\boldsymbol{i}}\right)_{\boldsymbol{i} \in \mathbb{Z}^{2}}$ be defined with the same Rademacher value for each component of $\boldsymbol{\Pi}_{\mu_{1}, \mu_{2}}$, independently from one component to another.

Theorem 2.3 (Th. 3.3 in [47]). For $S_{\boldsymbol{n}}=\sum_{\boldsymbol{i} \in R_{\boldsymbol{n}}} X_{\boldsymbol{i}}$, we have

$$
\left(\frac{S_{\lfloor\boldsymbol{n} \cdot \boldsymbol{t}\rfloor}}{n_{1}^{\alpha_{1}+1 / 2} n_{2}^{\alpha_{2}+1 / 2} \ell_{1}\left(n_{1}\right)^{-1} \ell_{2}\left(n_{2}\right)^{-1}}\right)_{\boldsymbol{t} \in[0,1]^{2}} \stackrel{J_{1}}{\Longrightarrow} \sigma_{1} \sigma_{2}\left(\mathbb{B}_{\boldsymbol{t}}^{(\boldsymbol{H})}\right)_{\boldsymbol{t} \in[0,1]^{2}} \quad \text { in } \mathbb{D}\left([0,1]^{2}\right)
$$

when $\min \left(n_{1}, n_{2}\right) \rightarrow+\infty$, with $\mathbb{B}^{(\boldsymbol{H})}$ the fractional Brownian sheet with parameter $\boldsymbol{H}=\left(\alpha_{1}+1 / 2, \alpha_{2}+1 / 2\right)$ and $\sigma_{i}$ defined in $(31), i=1,2$.

The proof of this two-dimensional version is much more involved than its one-dimensional counterpart for Theorem 2.1. Because of the product structure of the partition, unlike for the preceding two-dimensional model in Section 2.2.1, one cannot expect a representation as a linear field with martingale-difference innovations in the lexicographical order. Instead, the proof uses a representation of the field as a linear process in only one direction, that is of the form

$$
X_{\boldsymbol{k}}=\sum_{j_{1} \in \mathbb{Z}} q_{k_{1}-j_{1}}^{(1)} X_{\left(j_{1}, k_{2}\right)}^{*}, \quad \boldsymbol{k} \in \mathbb{Z}^{2}
$$

where, for each $j_{2} \in \mathbb{Z},\left(X_{j}^{*}\right)_{j_{1} \in \mathbb{Z}}$ is a one-dimensional martingale-difference sequence with respect to the filtration $\left(\sigma\left(X_{i}: i_{1}<j_{1}, i_{2} \in \mathbb{Z}\right)\right)_{j_{1} \in \mathbb{Z}}$ and $q_{k}^{(1)}=\mathbb{P}\left(0 \in A_{k}^{(1)}\right), k \in \mathbb{Z}$, where $A_{k}^{(1)}$ is the ancestral lines of $k$, related to the partition $\Pi_{\mu_{1}}$ like in Section 2.1 .

A new difficulty arises from the fact that the martingale differences are dependent via the Hammond-Sheffield partition $\Pi_{\mu_{2}}$ in the second direction. This difficulty can be overcome with the help of a decoupling argument introduced in the proof of Lemma 3.11 in [47].

Remark 2.3. This simple model can be extended to obtain invariance principles for any fractional Brownian sheet (i.e. for any $\left.\boldsymbol{H} \in(0,1)^{2}\right)$ by using a Karlin partition (see Remark 2.1) for directions with $H_{i} \in(0,1 / 2)$ or the finest partition (consisting in singletons) when $H_{i}=1 / 2$.

\section{Asymptotic SHAPE THEOREMS FOR RANDOM GROWTH MODELS}

This section presents some limit theorems for explicit discrete models and shows how they allow to get a precise insight into the behaviour of these models. The models we are interested in are random growth models, 
appearing typically in statistical mechanics or in the modeling of biological phenomena, and we give asymptotic shape theorems for their growth. The classical setting is to consider a graph $G=(V, E)$ with a distinguished vertex $O$, here $V$ stands for the set of vertices while $E$ is the set of edges. Adopting a biomedical point of view, we imagine an infection starting from $O$ and spreading through the edges according to some rules, involving randomness, in such a way that each vertex of the graph is eventually infected. Denoting by $T(x)$ the time required to infect a vertex $x \in V$, we are interested in the set $B(t)=\{x \in V: T(x) \leq t\}$ of infected vertices at time $t$. The term random growth model comes from the fact that the quantity $B(t)$ is increasing with respect to the time. A natural goal is to study the propagation of the infection by investigating the asymptotic form of the set $B(t)$ and to show that, renormalized by time $t, B(t)$ has a deterministic asymptotic shape when $t \rightarrow+\infty$. Such asymptotic shape theorem is a limit theorem of LLN type for the random set $B(t)$. In dimension one, it can be completed by a CLT result exhibiting Gaussian fluctuations of order $\sqrt{t}$ while, in higher dimension, the study of fluctuations are still open questions and it is conjectured that several of these models belong to the KPZ class, which is a family of models satisfying some specific scaling relations, in particular their orders of fluctuations differ from the typical $\sqrt{t}$ of the CLT, see for instance [33].

In the following, we will focus on processes on the graph $\mathbb{Z}^{d}$ but the questions are also very interesting in general graphs: in [94], Pemantle worked on trees; in [49-51], Durrett and its coauthors worked on finite networks; and more recently Mourrat and Valesin worked on finite random graphs in [88]. The question on infinite random graphs is still challenging, see [31] and [8] for contact process on the percolation cluster and [105] on the Boolean percolation cluster. Section 3.1 deals with the first passage percolation model, Section 3.2 with the contact process and Section 3.3 with non-attractive models.

\subsection{First Passage Percolation}

Random growth models were first introduced by Eden in 1961 on the graph $\mathbb{Z}^{2}$ and for discrete time. Next, they were extended by Hammersley and Welsh in 1965 on the graph $\mathbb{Z}^{d}$ and to general continuous infection time. This model is called First Passage Percolation (FPP) and is precisely defined as follows. On each edge $e$ of $\mathbb{Z}^{d}$, the random variable $t_{e}$ represents the passage time of the edge $e$, that is, the amount of time taken by the infection to cross the edge $e$. The collection $\left(t_{e}\right)$ is assumed to be iid nonnegative random variables. For two sites $x, y$ of $\mathbb{Z}^{d}$, the passage time $T(x, y)$ from $x$ to $y$ is defined as the infimum, over every path $\Gamma$ going from $x$ to $y$, of the quantity $\sum_{e \in \Gamma} t_{e}$. Many statements of asymptotic shape theorem exist for this FPP model with more or less restrictive assumptions, see for instance [107], [77], [15], [119], [74]. We give here one such result:

Theorem 3.1 (An asymptotic shape theorem for FPP). Let $\left(t_{i}\right)$ be iid copies of $t_{e}$ such that

(1) $\mathbb{E}\left[\min \left(t_{1}^{d}, \ldots, t_{2 d}^{d}\right)\right]<+\infty$,

(2) $\mathbb{P}\left(t_{e}=0\right)<p_{c}$ (critical point of the bond percolation on $\mathbb{Z}^{d}$ ).

Then, there exists a deterministic convex compact set $\mathcal{B}$ in $\mathbb{R}^{d}$ such that for each $\varepsilon>0$,

$$
\mathbb{P}\left((1-\varepsilon) \mathcal{B} \subset \frac{B(t)}{t} \subset(1+\varepsilon) \mathcal{B}, \text { for all large } t\right)=1
$$

This theorem is due to Cox and Durrett [32] but has benefited a lot from the seminal work of Richardson [107]. The strategy to prove this theorem is the following one:

- The first step is to prove a radial convergence, that is the convergence of the quantity $\frac{T(0, n x)}{n}$ as $n$ goes to infinity for any fixed direction $x \in \mathbb{Z}^{d}$. To do that, Kingman's subadditive ergodic theorem [79] ensures the convergence of this quantity under subadditivity (satisfied by definition of the hitting time), stationarity hypotheses (ensured by the iid environment) and integrability (following from Assumption (1)). For each $x \in \mathbb{Z}^{d}$, let $\mu(x)$ denote the limit of $\frac{T(0, n x)}{n}$. At this point, the growth of the ball $B(t)$ is proved to be at least linear.

- The second step is to extend the quantity $\mu(x)$ from $x \in \mathbb{Z}^{d}$ to $x \in \mathbb{R}^{d}$. It is easy to see that $\mu$ is a seminorm. Using Assumption (2), edges $e$ with infection cost $t_{e}=0$ are not too numerous and one can 
show that $\mu(x)>0$ so that $\mu$ can be extended as a norm on $\mathbb{R}^{d}$. In particular, the growth of the shape is at most linear.

- Knowing that the shape is blocked between two linear shapes, the exact linear growth still has to be proved. It comes from a uniform estimate of the different growth rates for close directions.

We refer to the recent survey [4] by Auffinger, Damron and Hanson for further results about the model of First Passage Percolation and corresponding present challenges.

\subsection{Contact processes}

We now consider models with possible extinction, that is, in our context of infection, a spontaneous recovery from the infection on each site is now allowed. The archetype is the contact process introduced by Harris in 1974 in [72]: this process is a continuous-time Markov process $\left(\eta_{t}\right)_{t \geq 0}$ with values in $\{0,1\}^{\mathbb{Z}^{d}}$ which splits up $\mathbb{Z}^{d}$ into "infected" sites $\left\{x \in \mathbb{Z}^{d}: \eta_{t}(x)=1\right\}$ and "healthy" sites $\left\{x \in \mathbb{Z}^{d}: \eta_{t}(x)=0\right\}$. Each infected site $x$ (i.e. $x \in \eta_{t}$ ) heals spontaneously (i.e. is removed from $\eta_{t}$ ) at rate 1 and each healthy site $x$ (i.e. $x \notin \eta_{t}$ ) gets infected (i.e. is added to $\eta_{t}$ ) at rate given by the number of its nearest neighbours $y$ that are infected at time $t$, multiplied by a parameter $\lambda>0$. This contact process is one of the simplest interacting particle systems that exhibit a phase transition. There exists a non-trivial critical value $0<\lambda_{c}<+\infty$ such that the probability that an infection starting from a single site propagates indefinitely is positive when $\lambda>\lambda_{c}$ and is zero when $\lambda\left\langle\lambda_{c}\right.$. We refer to [84] for background on such processes and related models.

In order to continue to deal with a random growth model, let consider the non decreasing family of set $B(t)=\left\{x \in \mathbb{Z}^{d}: T(x) \leq t\right\}$ where $T(x)$ is now the time of the first infection of the vertex $x$. The quantity $B(t)$ is no longer the set of infected sites at time $t$ but represents instead the set of sites which have been infected at least once before time $t$ (but maybe not infected anymore). Since the set $B(t)$ is constant after a certain time when the infection disappears, it is necessary, for the study to be relevant, to take $\lambda>\lambda_{c}$ and to work under the probability conditioned to the survival event $\mathscr{S}=\left\{\forall t>0, \exists x \in \mathbb{Z}^{d}\right.$ such that $\left.\eta_{t}(x)=1\right\}$. The following theorem can be credited to Durrett [48] but it is the result of various contributions involving several co-authors.

Theorem 3.2 (Asymptotic shape theorem for the contact process). For $\lambda>\lambda_{c}$, there exists a deterministic convex compact set $\mathcal{B}$ in $\mathbb{R}^{d}$ such that for each $\varepsilon>0$ :

$$
\mathbb{P}\left((1-\varepsilon) \mathcal{B} \subset \frac{B(t)}{t} \subset(1+\varepsilon) \mathcal{B}, \text { for all large } t \mid \mathscr{S}\right)=1 .
$$

There exists a lot of extensions of the classical contact process: for instance, a random environment affecting the infection rate (see [114], [57]), or the presence of intermediate sterile states which are not able to propagate the infection can be considered (see [80], [39]).

In any case, the proof is typically divided into the following distinct parts:

- The first step is to prove that, as soon as the process survives, the growth of $B(t)$ is at least linear. This step is difficult due to the fact that the extinction could slow down the process. In the case of the classical contact process, Bezuidenhout and Grimmet show in [9] that a supercritical process, conditioned to survive, stochastically dominates a two-dimensional supercritical oriented percolation. The authors of the various extensions then resorted to similar constructions, see [39,57, 114]. As a consequence, the required growth controls follow from this construction (and an additional by-product of the percolation construction, that is the fact that the process dies out on the critical region).

- By definition of the process, the "at most linear" part is obvious because in the Markov dynamics a site cannot be infected instantaneously (it would correspond to $\mathbb{P}\left(t_{e}=0\right)=0$ in the FPP model of Section 3.1).

- Like for the FPP model, the last step consists in proving the convergence of the renormalized set $B(t) / t$. In non-permanent models, the extinction is possible and the hitting times can be infinite, so that the standard integrability conditions are not satisfied. On the other hand, conditioning the model to survive can compromise stationarity and subadditivity properties. To overcome such lacks, another 
time quantity, denoted by $\theta(x)$, is considered. Heuristically, $\theta(x)$ represents a particular time when the site $x$ is infected and has infinitely many infected descendants. Obviously, $\theta(x)$ is bigger than the first infection time $T(x)$. Moreover, it turns out that this function $\theta$ satisfies adequate stationarity properties as well as the almost-subaddivity conditions involved in Kesten and Hammersley's theorem (see [70]), a well-known extension of Kingman's seminal result in [79]. The last part of the proof is then devoted to control the difference between $\theta$ and $T$, and this allows to transport the shape theorem from $\theta$ to the expected shape theorem for $T$. This strategy of proof is implemented in [40] for an extension of the classical contact process.

\subsection{Non-attractive models}

Despite its non-permanent behavior, the contact process is attractive in the sense that the more there are infected sites in the initial condition, the more the process has infected sites all the way long. Such a property is essential in the usual argument sketched above. However, some very famous interacting particle systems, like the kinetically constrained models (KCM), do not have this attractivity property so that all the subadditive methods fall down and the proof of linear velocity (LLN in dimension 1, asymptotic shape theorems in higher dimension) remains very challenging for such models.

KCM are interacting particle systems on $\mathbb{Z}^{d}$ which have been introduced in the physics literature in the ' $80 \mathrm{~s}$ (see [106] for a review) to model the liquid glass transition, a major open problem in condensed matter physics. A configuration of a KCM is given by assigning to each vertex $x \in \mathbb{Z}^{d}$ an occupation variable which corresponds to an empty or occupied site. The evolution is then given by a Markovian stochastic dynamics of Glauber type. With rate one, each vertex updates its occupation variable to occupied or to empty with probability $p \in[0,1]$ and $q=1-p$ respectively, if the configuration satisfies a certain local constraint. For the Fredrickson-Andersen one spin facilitated model (FA-1f), the constraint requires at least one empty nearest neighbour. Due to the presence of the constraint, FA-1f dynamic is not attractive.

In [14], FA-1f model is considered on $\mathbb{Z}$ starting from a configuration which has an empty site at the origin and is completely occupied in the left half line and the evolution of the front, namely the position of the leftmost empty site, is investigated. The idea explored in [14] is to couple FA-1f dynamics with a contact process (where, in this setting, empty sites correspond to infected sites) in order to use the well-known behavior of the contact process (ballistic motion of the front, shape theorem for the coupled zone) to study the FA-1f dynamics. Due to the fact that there is no constraint for the contact process for the move "empty" to "occupied" but there is one such constraint for the FA-1f, we can couple FA-1f and contact trajectories in such a way that FA-1f configurations contain more empty sites than contact process configurations. Such coupling construction, joined to the techniques of [13] to prove relaxation to equilibrium, allows to obtain the convergence to an invariant measure of the law of the process seen from the front. Then analyzing the increments of the front, a LLN is deduced. Finally, we obtain Gaussian fluctuations by generalizing the strategy of [56] based on Bolthausen's CLT in [16] for non stationary random variables under proper mixing condition.

Denoting $L O_{0}$ the subspace of initial configurations with a leftmost empty site at the origin, $\sigma_{t}$ the configurations of the FA-1f model at time $t$, and $X\left(\sigma_{t}\right)$ the front of $\sigma_{t}$, the LLN and the CLT state as follows:

Theorem 3.3. Let $q>\bar{q}$. There exist $s=s(q)$ and $v=v(q)$ such that for all $\sigma \in L O_{0}$ when $t \rightarrow+\infty$

$$
\begin{aligned}
\frac{X\left(\sigma_{t}\right)}{t} & \longrightarrow v \mathbb{P}_{\sigma} \text {-almost surely, } \\
\frac{X\left(\sigma_{t}\right)-v t}{\sqrt{t}} & \Longrightarrow \mathcal{N}\left(0, s^{2}\right) \quad \text { with respect to } \mathbb{P}_{\sigma} .
\end{aligned}
$$

Note that these asymptotics holds for $q>\bar{q}$ where $\bar{q}$ is related to the critical parameter $\lambda_{c}$ of the contact process, see just above Theorem 3.2. 
The motion of the front has also been analyzed in $[12,56]$ for another one dimensional KCM, the East model, for which the constraint requires the site at the right of $x$ to be empty: ergodicity of the measure seen from the front, LLN, CLT and cutoff results have been established.

Unfortunately, these techniques cannot be easily generalized in dimension $d \geq 2$. As for the contact process, for $q$ larger than some threshold, we can ensure the growth of the empty sites is of linear order but the techniques described above to prove the exact linear growth (subadditive methods, relaxation to equilibrium) are not directly translatable and shape theorems for kinetically constrained models are still open questions in higher dimensions, see Figure 4.

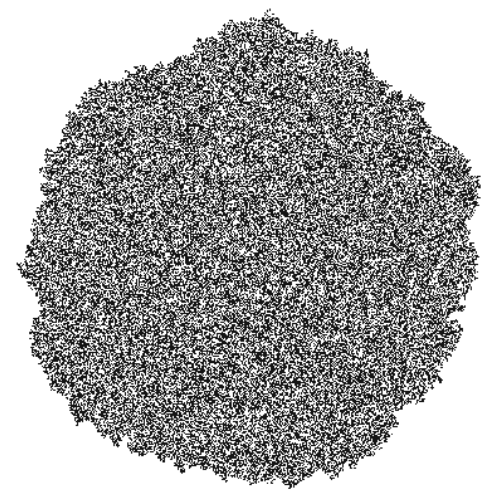

FIGURE 4. Illustration of a shape of infected points in FA-1f model.

\section{REFERENCES}

[1] B. Arras, E. Azmoodeh, G. Poly and Y. Swan. Stein Characterizations for Linear Combinations of Gamma Random Variables. To appear in Braz. J. Probab. Stat., 2019.

[2] B. Arras and C. Houdré. On Stein's Method for Infinitely Divisible Laws with Finite First Moment. To appear in SpringerBriefs in Probability and Mathematical Statistics.

[3] R. Arratia, A.D. Barbour and S. Tavaré. Logarithmic Combinatorial Structures: a Probabilistic Approach. European Mathematical Society, 2003.

[4] A. Auffinger, M. Damron and J. Hanson. 50 Years of First-Passage Percolation. American Mathematical Society. 2017.

[5] A.D. Barbour. Stein's Method for Diffusion Approximations. Probab. Theory Relat. Fields. 84(3), 297-322, 1990.

[6] A.D. Barbour, L.H.Y. Chen and W.-L. Loh. Compound Poisson Approximations for Nonnegative Random Variables Via Stein's Method. Ann. Probab. 20(4):1843-1866, 1992.

[7] A.D. Barbour and L.H.Y. Chen. An introduction to Stein's method. Lecture Notes Series Inst. Math. Sci. Natl. Univ. Singap., 4, Singapore University Press, Singapore, 2005.

[8] D. Bertacchi, N. Lanchier and F. Zucca. Contact and voter processes on the infinite percolation cluster as models of hostsymbiont interactions. Ann. Appl. Probab., 21(4):1215-1252, 2011.

[9] C. Bezuidenhout and G. Grimmett. The critical contact process dies out. Ann. Probab., 18(4):1462-1482, 1990.

[10] H. Biermé, O. Durieu and Y. Wang. Invariance principles for operator-scaling Gaussian random fields. Ann. Appl. Probab., 27(2):1190-1234, 2017.

[11] P. Billingsley. Convergence of probability measures. Wiley series in Probability and Statistics: Probability and Statistics, 2nd Edition, 1999.

[12] O. Blondel. Front progression in the East model. Stochastic Process. Appl., 123(9):3430-3465, 2013.

[13] O. Blondel, N. Cancrini, F. Martinelli, C. Roberto and C. Toninelli. Fredrickson-Andersen one spin facilitated model out of equilibrium. Markov Process. Related Fields, 19(3):383-406, 2013.

[14] O. Blondel, A. Deshayes and C. Toninelli. Front evolution of the Fredrickson-Andersen one spin facilitated model. Electronic Journal of Probability. 24:1-32, 2019.

[15] D. Boivin. First passage percolation: the stationary case. Probab. Theory Related Fields, 4:491-499, 1990.

[16] E. Bolthausen. On the central limit theorem for stationary mixing random fields. Ann. Probab., 10(4):1047-1050, 1982.

[17] E. Bolthausen. An estimate of the remainder in a combinatorial central limit theorem. Z. Wahrsch. Verw. Gebiete, 66(3):379$386,1984$. 
[18] R.C. Bradley. Introduction to strong mixing conditions. Kendrick Press, Heber City, UT, Vol. 1-3, 2007.

[19] J.-C. Breton, A. Clarenne and R. Gobard. Macroscopic analysis of determinantal random balls. Bernoulli, 25(2):1568-1601, 2019.

[20] J.-C. Breton and I. Nourdin. Error bounds on the non-normal approximation of Hermite power variations of fractional Brownian motion. Electron. Commun. Probab., 13:48-493, 2008.

[21] P. Breuer and P. Major. Central limit theorems for nonlinear functionals of Gaussian fields. J. Multivariate Anal., 13(3):425441, 1983.

[22] T.C. Brown and M.J. Phillips. Negative Binomial Approximations with Stein's Method. Methodol. Comput. Appl. Probab. 1(4): 407-421, 1999.

[23] F. Cellarosi and Y. G. Sinai. Non-Standard Limit Theorems in Number Theory. Prokhorov and contemporary probability theory, Springer Berlin, 197-213, 2013.

[24] S. Chatterjee. A New Method of Normal Approximation. Ann. Probab., 36(4):1584-1610, 2008.

[25] S. Chatterjee. Fluctuations of eigenvalues and second order Poincaré Inequalities. Probab. Theory Relat. Fields 143:1-40, 2009.

[26] S. Chatterjee. A short survey of Stein's method. Proceedings of the ICM, IV: 1-24, Seoul 2014.

[27] S. Chatterjee, J. Fulman and A. Röllin. Exponential Approximation by Stein's Method and Spectral Graph Theory. Lat. Am. J. Probab. Math. Stat. 8:197-223, 2011.

[28] L.H.Y. Chen. Poisson Approximation for Dependent Trials. Ann. Probab. 3(3):534-545, 1975.

[29] L.H.Y. Chen and S.T. Ho. An $L^{p}$ bound for the remainder in a combinatorial central limit theorem. Ann. Probability, 6(2):231-249, 1978.

[30] L.H.Y. Chen, L. Goldstein and Q.M. Shao. Normal Approximation by Stein's Method. Probability and its Application, Springer, Heidelberg, 2011.

[31] X. Chen and Q. Yao. The complete convergence theorem holds for contact processes on open clusters of $\mathbb{Z}^{d} \times \mathbb{Z}_{+}$. J. Stat. Phys., 135(4):651-680, 2009.

[32] J.T. Cox and R. Durrett. Some Limit Theorems for Percolation Processes with Necessary and Sufficient Conditions. Ann. Probab., 9(4):583-603, 1981.

[33] M. Damron. Random growth models: shape and convergence rate. Random growth models, 1-37, Proc. Sympos. Appl. Math., 75, Amer. Math. Soc., Providence, RI, 2018.

[34] Y.A. Davydov. The invariance principle for stationary processes. Teor. Verojatnost. i Primenen., 15:498-509, 1970.

[35] L. Decreusefond. The Stein-Dirichlet-Malliavin method. Modélisation Aléatoire et Statistique-Journées MAS 2014. ESAIM Proc. Surveys 51:49-59, 2015.

[36] J. Dedecker. A central limit theorem for stationary random fields. Probab. Theory Related Fields, 110(3):397-426, 1998.

[37] J. Dedecker, P. Doukhan, G. Lang, J.R. León, S. Louhichi and C. Prieur. Weak dependence: with examples and applications. Lecture Notes in Statistics, 190, Springer, New York, 2007.

[38] J. Dedecker, F. Merlevède and M. Peligrad. Invariance principles for linear processes with application to isotonic regression. Bernoulli, 17 (1):88-113, 2011.

[39] A. Deshayes. The contact process with aging. ALEA Lat. Am. J. Probab. Math. Stat., 11:845-883, 2014

[40] A. Deshayes. An asymptotic shape theorem for random linear growth models. arXiv:1505.05000, 2015.

[41] K. Dickman. On the frequency of numbers containing prime factors of a certain relative magnitude. Ark. Mat. Astronomi och Fysik, 22:1-14, 1930.

[42] C. Döbler. Stein's method of exchangeable pairs for the beta distribution and generalizations. Electron. J. Probab., 20(109):1$34,2015$.

[43] C. Döbler and G. Peccati. The Gamma Stein equation and noncentral de Jong theorems. Bernoulli, 24(4B):3384-3421, 2018.

[44] R.L. Dobrushin and P. Major. Non-central limit theorems for nonlinear functionals of Gaussian fields. Z. Wahrsch. Verw. Gebiete, 50:(1)27-52, 1979.

[45] M.D. Donsker. An invariance principle for certain probability limit theorems. Mem. Amer. Math. Soc., 6, 1951.

[46] O. Durieu and Y. Wang. From infinite urn schemes to decompositions of self-similar Gaussian process. Electron. J. Probab., 21: 1083-6489, 2016.

[47] O. Durieu and Y. Wang. From random partitions to fractional Brownian sheets. Bernoulli, 25(2):1412-1450, 2019.

[48] R. Durrett. The Contact Process, 1974-1989. Lectures in Applied Mathematics, Amer. Math. Soc., 27:1-18, 1991.

[49] R. Durrett and X.F. Liu. The contact process on a finite set I. Ann. Probab., 16(3):1158-1173, 1988.

[50] R. Durrett and R.H. Schonmann. The contact process on a finite set II. Ann. Probab., 16(4):1570-1583, 1988.

[51] R. Durrett, R.H. Schonmann and N.I. Tanaka. The contact process on a finite set III. Ann. Probab., 17(4):1303-1321, 1989.

[52] M. El Machkouri, D. Volný and W.B. Wu. A central limit theorem for stationary random fields. Stochastic Process. Appl., 123(1):1-14, 2013.

[53] N. Enriquez. A simple construction of the fractional Brownian motion. Stochastic Process. Appl., 109(2):203-223, 2004.

[54] C.-G. Esseen. Fourier analysis of distribution functions. A mathematical study of the Laplace-Gaussian law. Acta Math. $77: 1-125,1945$ 
[55] J. Fulman and N. Ross. Exponential Approximation and Stein's Method of Exchangeable Pairs. ALEA Lat. Am. J. Probab. Math. Stat. 10(1):1-13, 2013.

[56] S. Ganguly, E. Lubetzky and F. Martinelli. Cutoff for the east process. Comm. Math. Phys., 335(3):1287-1322, 2015.

[57] O. Garet and R. Marchand. Asymptotic shape for the contact process in random environment. Ann. Appl. Probab, 22(4):13621410, 2012.

[58] R.E. Gaunt. Variance-gamma approximation via Stein's method. Electron. J. Probab., 19(38):1-33, 2014.

[59] R.E. Gaunt. A Stein characterisation of the generalized hyperbolic distribution. ESAIM Probab. Stat., 21:303-316, 2017.

[60] R.E. Gaunt. On Stein's method for products of normal random variables and zero bias couplings. Bernoulli, 23:3311-3345, 2017.

[61] R.E. Gaunt. Products of normal, beta and gamma random variables: Stein operators and distributional theory. Braz. J. Probab. Stat., 32:437-466, 2018.

[62] R.E. Gaunt. Wasserstein and Kolmogorov Error Bounds for Variance-Gamma Approximation via Stein's Method I. J. Theoret. Probab., 1-41, 2018.

[63] R.E. Gaunt. Stein operators for variables from the third and fourth Wiener chaoses. Statist. Probab. Lett., 145:118-126, 2019.

[64] R.E. Gaunt, A.M. Pickett and G. Reinert. Chi-square approximation by Stein's method with application to Pearson's statistic. Ann. Appl. Probab. 27(2):720-756, 2017.

[65] R.E. Gaunt, G. Mijoule and Y. Swan. An algebra of Stein operators. J. Math. Anal. Appl., 469:260-279, 2019.

[66] L. Giraitis and D. Surgailis. CLT and other limit theorems for functionals of Gaussian processes. Z. Wahrsch. Verw. Gebiete, 70(2):191-212, 1985.

[67] L. Goldstein and G. Reinert. Stein's method for the Beta distribution and the Pólya-Eggenberger urn. J. Appl. Probab., 50:1187-1205, 2013.

[68] F. Götze. On the Rate of Convergence in the Multivariate CLT. Ann. Probab. 19(2):724-739, 1991.

[69] F. Götze and A.N. Tikhomirov. Limit theorems for spectra of random matrices with martingale structure. Teor. Veroyatnost. i Primenen., 51:171-192, 2006.

[70] J.M. Hammersley. Postulates for subadditive processes. Ann. Probability, 2:652-680, 1974.

[71] A. Hammond and S. Sheffield. Power law Pólya's urn and fractional Brownian motion. Probab. Theory Related Fields, 157 (1-3):691-719, 2013.

[72] T.E. Harris. Contact interactions on a lattice. Ann. Probability, 2:969-988, 1974.

[73] C. Houdré, V. Pérez-Abreu and D. Surgailis. Interpolation, correlation identities and inequalities for infinitely divisible variables. J. Fourier Anal. Appl. 4(6):651-668, 1998.

[74] C.D. Howard. Models of first-passage percolation. Probability on discrete structures, 110:125-173, 2004.

[75] Z.J. Jurek. On relations between Urbanik and Mehler semigroups. Probability and Mathematical statistics, 29(2):297-308, 2009.

[76] S. Karlin. Central limit theorems for certain infinite urn schemes. J. Math. Mech., 17:373-401, 1967.

[77] H. Kesten. Aspects of first passage percolation. École d'été de probabilités de Saint-Flour, XIV-1984, 125-264, 1986.

[78] A.Y. Khintchine. Zur Theorie der unbeschränkt teilbaren Verteilungsgesetze. Mat. Sbornik, 44(1):79-119, 1937.

[79] J.F.C. Kingman. Subadditive ergodic theory. Ann. Probability, 1:883-909, 1973.

[80] S.M. Krone. The two-stage contact process. Ann. Appl. Probab., 9(2):331-351, 1999.

[81] R. Lachièze-Rey, M. Schulte and J.E. Yukich. Normal approximation for stabilizing functionals. Ann. Appl. Probab., 29(2):931993, 2019.

[82] G. Last, G. Peccati and M. Schulte. Normal approximation on poisson spaces: Mehler's formula, second order Poincaré inequalities and stabilization. Probability theory and related fields, 165(3-4):667-723, 2016.

[83] C. Ley, G. Reinert and Y. Swan. Stein's method for comparison of univariate distributions. Probab. Surveys, 14:1-52, 2017.

[84] T.M. Liggett. Interacting particle systems. Classics in Mathematics. Springer-Verlag, Berlin, 2005.

[85] H.M. Luk. Stein's method for the Gamma distribution and related statistical applications. Ph.D thesis, University of Southern California, 1994.

[86] I.W. McKeague, E. Peköz and Y. Swan. Stein's method and approximating the quantum harmonic oscillator. Bernoulli, 25(1):89-111, 2019.

[87] F. Merlevède, M. Peligrad and S. Utev. Recent advances in invariance principles for stationary sequences. Probab. Surv., $3: 1-36,2006$.

[88] J.-C. Mourrat and D. Valesin. Phase transition of the contact process on random regular graphs. Electron. J. Probab., 21:No. 31, 17 pp., 2016.

[89] I. Nourdin and G. Peccati. Stein's method on Wiener chaos. Probab. Theory Related Fields 145(1-2):75-118, 2009.

[90] I. Nourdin and G. Peccati. Normal approximations with Malliavin calculus. From Stein's method to universality. Cambridge Tracts in Mathematics, 192. Cambridge University Press, Cambridge, 2012.

[91] G. Peccati, J.L. Solé, M.S. Taqqu and F. Utzet. Stein's method and normal approximation of Poisson functionals. Ann. Prob., 38:443-478, 2010.

[92] G. Peccati. Quantitative CLTs on a Gaussian space: a survey of recent developments. Journées MAS 2012, ESAIM Proc., 44:61-78, 2014. 
[93] E. A. Peköz and A. Röllin. New Rates for Exponential Approximation and the Theorems of Rényi and Yaglom. Ann. Probab. 39(2), 587-608, 2001.

[94] R. Pemantle. The contact process on trees. Ann. Probab., 20(4):2089-2116, 1992.

[95] M.D. Penrose and J.E. Yukich. Central limit theorems for some graphs in computational geometry. Ann. Appl. Probab., 11:1005-1041, 2011.

[96] V.V. Petrov. Limit theorems of probability theory. Sequences of independent random variables. Oxford Studies in Probability, vol. 4. Oxford Science Publications, Oxford University Press, 1995.

[97] A. Pickett. Rates of convergence of Chi-square approximations via Stein's method. Ph.D. thesis, University of Oxford, 2004.

[98] J. Pike and H. Ren. Stein's Method and the Laplace Distribution. ALEA Lat. Am. J. Probab. Math. Stat., 11(1):571-587, 2014.

[99] V. Pilipauskaite and D. Surgailis. Anisotropic scaling of the random grain model with application to network traffic. J. Appl. Probab., 53(3):857-879, 2016.

[100] V. Pilipauskaitè and D. Surgailis. Scaling transition for nonlinear random fields with long-range dependence. Stochastic Process. Appl., 127(8):2751-2279, 2017.

[101] V. Pipiras and M.S. Taqqu. Long-range dependence and self-similarity. Cambridge Series in Statistical and Probabilistic Mathematics, (45) 2017.

[102] N. Privault. Stochastic Analysis in Discrete and Continuous Settings with Normal Martingales. Springer, Berlin, 2009.

[103] D. Puplinskaite and D. Surgailis. Aggregation of autoregressive random fields and anisotropic long-range dependence. Bernoulli, 22(4):2401-2441, 2016.

[104] M. Reitzner. Central limit theorems for random polytopes. Probab. Theory Related Fields, 133:488-507, 2005.

[105] T. Riblet. Contact process on subcritical boolean percolation cluster. Work in progress, http://www.iecl.univlorraine.fr/ Tom.Riblet/, 2019+.

[106] F. Ritort and P. Sollich. Glassy dynamics of kinetically constrained models. Advances in Physics, 52(4):219-342, 2003.

[107] D. Richardson. Random growth in a tessellation. Proc. Cambridge Philos. Soc., 74:515-528, 1973.

[108] M. Rosenblatt. Invariant and subinvariant measures of transition probability functions acting on continuous functions. $Z$. Wahrsch. Verw. Gebiete, 25:209-221, 1972/73.

[109] N.F. Ross. Fundamentals of Stein's method. Probab. Surv. 8:210-293, 2011.

[110] G. Samorodnitsky. Stochastic processes and long range dependence. Springer Series in Operations Research and Financial Engineering, 2016.

[111] G. Samorodnitsky and M.S. Taqqu. Stable non-Gaussian random processes. Stochastic Modeling, Chapman \& Hall, New York, 1994.

[112] K. Sato. Lévy processes and infinitely divisible distributions. Cambridge University Press, 2015.

[113] Y. Shen and Y. Wang. Operator-scaling Gaussian random fields via aggregation. arXiv:1712.07082, 2017.

[114] J. E. Steif and M. Warfheimer. The critical contact process in a randomly evolving environment dies out. ALEA Lat. Am. J. Probab. Math. Stat., 4:337-357, 2008.

[115] C. Stein. A bound for the error in the normal approximation to the distribution of a sum of dependent random variables. Proceedings of the Sixth Berkeley Symposium on Mathematical Statistics and Probability, 583-602, 1972.

[116] C. Stein. Approximate computation of expectations. Institute of Mathematical Statistics Lecture Notes Monograph Series, 7 , 1986.

[117] M.S. Taqqu. Weak convergence to fractional Brownian motion and to the Rosenblatt process. Z. Wahrsch. Verw. Gebiete, 31: 287-302, 1974/75.

[118] M.S. Taqqu. Convergence of integrated processes of arbitrary Hermite rank. Z. Wahrsch. Verw. Gebiete 50:(1):5-83, 1979.

[119] M.Q. Vahidi-Asl and J.C. Wierman. A shape result for first-passage percolation on the Voronoï tessellation and Delaunay triangulation. Random graphs, Vol. 2 (Poznań, 1989), 247-262, 1992.

[120] D. Volný. A central limit theorem for fields of martingale differences. C. R. Math. Acad. Sci. Paris, 353(12):1159-1163, 2015.

[121] M.J. Wichura. Inequalities with applications to the weak convergence of random processes with multi-dimensional time parameters. Ann. Math. Statist., 40:681-687, 1969.

[122] L. Xu. Approximation of stable law in Wasserstein-1 distance by Stein's method. Ann. Appl. Probab., 29(1):458-504, 2019. 